\title{
LOCALLY FULL HNN EXTENSIONS OF INVERSE SEMIGROUPS
}

\author{
AKIHIRO YAMAMURA \\ (Received 1 May 1998; revised 25 October 2000) \\ Communicated by D. Easdown
}

\begin{abstract}
We investigate a locally full HNN extension of an inverse semigroup. A normal form theorem is obtained and applied to the word problem. We construct a tree and show that a maximal subgroup of a locally full HNN extension acts on the tree without inversion. Bass-Serre theory is employed to obtain a group presentation of the maximal subgroup as a fundamental group of a certain graph of groups associated with the $\mathscr{D}$-structure of the original semigroup.
\end{abstract}

2000 Mathematics subject classification: primary 20M18, 20M05, 20E08, $20 \mathrm{E} 06$.

Keywords and phrases: HNN extensions, Inverse semigroups, Normal form, Word problem, Bass-Serre theory.

\section{Introduction and preliminaries}

1.1. Background The study on the embeddability of HNN extensions of semigroups began in [7]. The HNN extension of a semigroup was formalized in terms of presentations, and several types of embeddings of semigroups into HNN extensions and the relationship with amalgamated free products were studied in $[15,16]$. Various inverse semigroups have an HN extension structure. For example, free inverse semigroups, free inverse monoids and free Clifford semigroups can be presented as an HNN extension of a semilattice. Furthermore, any Bruck-Reilly extension of an inverse monoid is an HNN extension of an appropriate inverse monoid, and hence, any bisimple regular $\omega$-semigroup and any simple regular $\omega$-semigroup can be presented as an HNN extension ([18]).

There exist considerable applications of HNN extensions to algorithmic and structural problems in inverse semigroup theory as well as in group theory. The undecidability of Markov properties and several other properties of inverse semigroups are

(c) 2001 Australian Mathematical Society $0263-6115 / 2001 \$ A 2.00+0.00$ 
proved using HNN extensions and amalgamated free products $([15,16])$. Furthermore, HNN extensions are applied to give an alternative proof of Reilly's theorem that every inverse semigroup can be embedded into a bisimple inverse monoid ([15]).

In [17], HNN extensions are employed to investigate an idempotent pure image of a free inverse semigroup and to prove the existence of an F-inverse cover over a free group for an inverse semigroup. There exist numerous similarities between an HNN extension of a semilattice and a free group. Therefore, an HNN extension of a semilattice is considered as a natural generalization of a free group. For example, an HNN extension of a semilattice is the universal object in a certain category on a fixed set of generators as a free group is the universal object of the class of groups generated by a fixed set. Contracted Schützenberger graphs of an HNN extension of a semilattice with respect to a certain subset form a forest. Recall that the Cayley graph of a free group with respect to its base is a tree. The Nielsen-Schreier subgroup theorem in group theory can be generalized to the class of full HNN extensions of semilattices with an identity.

These results suggest more applications of HNN extensions to algorithmic and structural problems in inverse semigroup theory and motivate us to study the structure of HNN extensions. An HNN extension is called locally full if the associated inverse monoids are full in the local monoids defined by their identities. This condition makes the structure of HNN extensions transparent. In fact, locally full HNN extensions resemble HNN extensions of groups and have many nice properties. Therefore, we study locally full HNN extensions in this paper.

Let us outline the paper. In this section, we review several fundamental results in inverse semigroup theory. In Section 2, we obtain the normal form theorem for a locally full HNN extension. In Section 3, we apply it to solve the word problem. In Section 4, we build a tree on which a maximal subgroup of a locally full HNN extension acts. In Section 5, the Bass-Serre theory is employed to obtain a group presentation for the maximal subgroup. In Section 6, we give several examples.

For basic results in inverse semigroup theory we refer the reader to $[9,13]$. We use basic results and terminologies from group theory without mention. The reader is referred to $[3,10,14]$ for definitions and results on combinatorial group theory.

\subsection{Presentations We briefly review inverse semigroup presentations and $\mathrm{HNN}$} extensions. A semigroup $S$ is called inverse if there exists a unique element $x^{-1}$ satisfying $x x^{-1} x=x$ and $x^{-1} x x^{-1}=x^{-1}$ for every $x$ in $S$. It is equivalent to say that $S$ is a regular semigroup (in the sense of von Neumann) whose idempotents form a semilattice. The set of idempotents of a semigroup $S$ is denoted by $E(S)$. The class of inverse semigroups has a free object, that is, there exists a free inverse semigroup on any non-empty set. Let $X$ be a non-empty set. Then the free semigroup on $X$ is just the set of all non-empty words on $X$ and is denoted by $X^{+}$. Now we 
take a copy $X^{-1}$ of $X$ such that $X^{-1}$ is disjoint from $X$. We have a one to one correspondence $^{-1}: X \rightarrow X^{-1}\left(x \rightarrow x^{-1}\right.$ for all $\left.x \in X\right)$. We extend the mapping ${ }^{-1}$ to the correspondence ${ }^{-1}:\left(X \cup X^{-1}\right) \rightarrow\left(X \cup X^{-1}\right)$ by defining $\left(x^{-1}\right)^{-1}=x$ for all $x^{-1} \in X^{-1}$. If $w \in\left(X \cup X^{-1}\right)^{+}$, then we can write $w=x_{1} x_{2} \cdots x_{n}$, where $x_{i} \in X \cup X^{-1}$ for every $i=1,2, \ldots, n$. We denote the element $x_{n}^{-1} x_{n-1}^{-1} \cdots x_{2}^{-1} x_{1}^{-1}$ by $w^{-1}$. Note that the operation ${ }^{-1}$ is an involution. Consider the relation $R_{0}$ consisting of all pairs of the form $\left(w w^{-1} w, w\right)$ and $\left(w w^{-1} z z^{-1}, z z^{-1} w w^{-1}\right)$, where $w$ and $z$ are elements of $\left(X \cup X^{-1}\right)^{+}$. The Wagner congruence $\theta$ is the congruence generated by $R_{0}$. Then the semigroup $\left(X \cup X^{-1}\right)^{+} / \theta$ is the free inverse semigroup on $X$. Denote the free inverse semigroup on $X$ by $\operatorname{FIS}(X)$.

Every inverse semigroup is a homomorphic image of a suitable free inverse semigroup; if $S$ is an inverse semigroup, there exists a certain non-empty set $X$ such that FIS $(X) / \eta=S$ for some congruence $\eta$ on FIS $(X)$. If $\eta$ is generated by some set $R$ of relations on $\left(X \cup X^{-1}\right)^{+}$, then we say that $S$ is presented by the set $X$ of generators and the set $R$ of relations. Then we write $S=\operatorname{Inv}(X \mid R)$. If $|X|$ is finite, $S$ is called finitely generated. If both $|X|$ and $|R|$ are finite, then $S$ is called finitely presented.

Assume that an inverse semigroup $S$ has a presentation $\operatorname{Inv}(X \mid R)$. Take a set $Y$ disjoint from $X \cup X^{-1}$ and the set $R^{\prime}$ of relations on $\left(Y \cup Y^{-1}\right)^{+}$. Then the inverse semigroup presented by $\operatorname{Inv}\left(X, Y \mid R, R^{\prime}\right)$ is denoted by $\operatorname{Inv}\left(S, Y \mid R^{\prime}\right)$ for brevity.

The inverse semigroup $\operatorname{Inv}(X \mid R)$ is regarded as the freest inverse semigroup generated by the set $X$ subject to the relation $R$ because of the following well-known result, which is equivalent to von Dyck's theorem in group theory.

PROPOSITION 1.1. Suppose that an inverse semigroup $S$ is presented by $\operatorname{Inv}(X \mid R)$ and $\phi$ is a homomorphism of FIS $(X)$ into an inverse semigroup T. If $\phi\left(w_{1}\right)=\phi\left(w_{2}\right)$ in $T$ for all $w_{1}, w_{2} \in\left(X \cup X^{-1}\right)^{+}$with $w_{1}=w_{2}$ in $R$, then there exists a homomorphism $\psi: S \rightarrow T$ such that $\phi(x)=\psi(\varphi(x))$ for every $x \in X$, where $\varphi$ is the natural homomorphism of $\operatorname{FIS}(X)$ into $S$.

1.3. HNN extensions and embeddability Let $S$ be an inverse semigroup, and let $A_{i}$ and $B_{i}(i \in I)$ be invers subsemigroups of $S$. Suppose that $e_{i} \in A_{i} \subset e_{i} S e_{i}$, $f_{i} \in B_{i} \subset f_{i} S f_{i}$ for some idempotents $e_{i}, f_{i}$ of $S$ and that $\phi_{i}: A_{i} \rightarrow B_{i}$ is an isomorphism for every $i \in I$. Then the inverse semigroup $S^{*}$ presented by

$$
\begin{aligned}
& \operatorname{Inv}\left(S, t_{i}(i \in I) \mid t_{i}^{-1} a t_{i}=\phi_{i}(a) \text { for every } a \in A_{i},\right. \\
& \left.t_{i}^{-1} t_{i}=f_{i}, t_{i} t_{i}^{-1}=e_{i} \text { for every } i \in I\right),
\end{aligned}
$$

or equivalently,

$$
\begin{aligned}
& \operatorname{Inv}\left(S, t_{i}(i \in I) \mid t_{i}^{-1} a t_{i}=\phi_{i}(a) \text { for every } a \in A_{i}^{\prime},\right. \\
& \left.\qquad t_{i}^{-1} t_{i}=f_{i}, t_{i} t_{i}^{-1}=e_{i} \text { for every } i \in I\right),
\end{aligned}
$$


where $A_{i}^{\prime}$ is a set of generators of $A_{i}$, is called the HNN extension of $S$ associated with $\phi_{i}: A_{i} \rightarrow B_{i}(i \in I)$. Each element $t_{i}$ in $S^{*}$ is called a stable letter. In general, a class $\mathbf{C}$ of semigroups is said to have the weak HNN property if $\mathbf{C}$ satisfies the following condition.

Suppose that $S, A, B \in \mathrm{C}, e \in A \subset e S e, f \in B \subset f S f$ for some $e, f \in E(S)$. Let $\phi: A \rightarrow B$ be an isomorphism. Then there exist $T \in \mathbf{C}$ and an embedding $\psi: S \hookrightarrow T$ such that

$$
t^{\prime} \psi(a) t=\psi(\phi(a)) \quad \text { for all } a \in A,
$$

and

$$
t^{\prime} t=\psi(f) \text { and } t t^{\prime}=\psi(e) \text { for some } t \in T \text { and its inverse } t^{\prime}
$$

A class $\mathbf{C}$ of semigroups is said to have the strong HNN property if $\mathbf{C}$ satisfies the following condition.

Suppose that $S, A, B \in \mathbf{C}$ and $A \subset e S e, B \subset f S f$ for some $e, f \in E(S)$. Let $\phi: A \rightarrow B$ be an isomorphism. Then there exist $T \in \mathbf{C}$ and an embedding $\psi: S \hookrightarrow T$ satisfying the conditions (1.3), (1.4) and

$$
t^{\prime} \psi(S) t \cap \psi(S)=t^{\prime} \psi(A) t=\psi(B)
$$

PROPOSITION $1.2([15,16])$. The class of inverse semigroups has the strong HNN property.

By Proposition 1.2, an inverse semigroup $S$ is always embedded into $S^{*}$ presented by (1.1). We usually identify $S$ with the corresponding inverse subsemigroup of the HNN extension under the natural isomorphism, and hence, (1.5) implies

$$
t_{i}^{-1} S t_{i} \cap S=t_{i}^{-1} A_{i} t_{i}=B_{i} \quad \text { for every } i \in I
$$

in the HNN extension.

We note that the class of inverse semigroups also has the strong amalgamation property $([5,6])$. The weak and strong HNN embeddability of several classes of semigroups and the relationship with the amalgamation property are investigated in $[15,16]$.

1.4. Locally full HNN extensions If $A_{i}$ is full in $e_{i} S e_{i}$ and $B_{i}$ is full in $f_{i} S f_{i}$, that is, $E\left(A_{i}\right)=E\left(e_{i} S e_{i}\right)$ and $E\left(B_{i}\right)=E\left(f_{i} S f_{i}\right)$ for every $i \in I$, then the HNN extension $S^{*}$ is called locally full. If $M$ is an inverse monoid and $A_{i}$ and $B_{i}$ are full submonoids, that is, $E\left(A_{i}\right)=E(M)$ and $E\left(B_{i}\right)=E(M)$, then the HNN extension $M^{*}$ is called full. Full HNN extensions of inverse monoids and locally full HNN extensions of semilattices are investigated in [15] and [17], respectively. 
For example, the free Clifford semigroup on the set $\left\{t_{1}, t_{2}, \ldots, t_{n}\right\}$ is presented by

$$
\begin{aligned}
& \operatorname{Inv}\left(E, t_{1}, t_{2}, \ldots, t_{n} \mid t_{i}^{-1} f t_{i}=\phi_{i}(f)=f \text { for every } f \in E e_{i},\right. \\
& \left.\qquad t_{i}^{-1} t_{i}=t_{i} t_{i}^{-1}=e_{i} \text { for every } i=1,2, \ldots n\right),
\end{aligned}
$$

where $E$ is the free semilattice on the set $X=\left\{e_{1}, e_{2}, \ldots, e_{n}\right\}$ and $\phi_{i}$ is the identity mapping of $E e_{i}$ for every $i=1,2, \ldots, n$. Hence, any free Clifford semigroup is a locally full $\mathrm{HNN}$ extension of a free semilattice.

The formation of a locally full HNN extension yields no new idempotents as we see in Corollary 3.1. Hence, the partial order structure of a locally full HNN extension is not so complicated as a more general HNN extension. We can generalize Britton's lemma to the class of locally full HNN extensions. The following is obtained in [17].

PROPOSITION 1.3. Any HNN extension of a semilattice $E$ with stable letters $t_{i}$ $(i \in I)$ can be presented as a locally full $\mathrm{HNN}$ extension of a semilattice $E_{0}$ with $|I|$ stable letters, where $E_{0}$ is an extension of $E$.

A similar result holds for $\mathrm{HNN}$ extensions of inverse semigroups.

PROPOSITION 1.4. Any HNN extension of an inverse semigroup $S$ with stable letters $t_{i}(i \in I)$ can be presented as a locally full $\mathrm{HNN}$ extension of $T_{0}$ with $|I|$ stable letters, where $T_{0}$ is an inverse semigroup extension of $S$.

PROOF. Let $S$ be an inverse semigroup. Suppose that $A_{i}$ and $B_{i}$ are inverse subsemigroups of $S$ such that $e_{i} \in A_{i} \subset e_{i} S e_{i}$ and $f_{i} \in B_{i} \subset f_{i} S f_{i}$ for some $e_{i}, f_{i} \in E(S)$ for every $i \in I$. Let $\phi_{i}$ be an isomorphism of $A_{i}$ onto $B_{i}$ for every $i \in I$. We show that the HNN extension $S^{*}$, presented by (1.1), is a locally full HNN extension of a certain inverse semigroup with the same cardinal number of stable letters. Let $T_{0}$ be an inverse subsemigroup of $S^{*}$ generated by $S$ and $E\left(S^{*}\right)$. Let $C_{i}$ be the inverse subsemigroup of $S^{*}$ generated by $A_{i}$ and $E\left(S^{*}\right) e_{i}$ for each $i \in I$. Let $D_{i}$ be the inverse subsemigroup of $S^{*}$ generated by $B_{i}$ and $E\left(S^{*}\right) f_{i}$ for each $i \in I$. We note that $C_{i}$ and $D_{i}$ are inverse subsemigroups of $S^{*}$ such that $e_{i} \in C_{i} \subset e_{i} S^{*} e_{i}$ and $f_{i} \in D_{i} \subset f_{i} S^{*} f_{i}$ for each $i \in I$. We also noth that $E\left(C_{i}\right)=E\left(e_{i} S^{*} e_{i}\right)$ and $E\left(D_{i}\right)=E\left(f_{i} S^{*} f_{i}\right)$ for each $i \in I$. We define a mapping $\phi_{i}^{*}$ of $C_{i}$ into $D_{i}$ by $\phi_{i}^{*}(s)=t_{i}^{-1} s t_{i}$ for $s \in C_{i}$. Since $t_{i}^{-1} A_{i} t_{i} \subset B_{i}$ and $t_{i}^{-1} E\left(S^{*}\right) e_{i} t_{i} \subset E\left(S^{*}\right) f_{i}$ for each $i \in I$, we have $t_{i}^{-1} C_{i} t_{i} \subset D_{i}$ for each $i \in I$. Hence, $\phi_{i}^{*}$ is well defined. Similarly, we can show that $t_{i} D_{i} t_{i}^{-1} \subset C_{i}$ for each $i \in I$. It is clear that $\phi_{i}^{*}$ is an isomorphism of $C_{i}$ onto $D_{i}$ for each $i \in I$ and that the restriction $\left.\phi_{i}^{*}\right|_{A_{i}}$ of $\phi_{i}^{*}$ to $A_{i}$ is equal to $\phi_{i}$. Let $T$ be the inverse semigroup presented by

$$
\begin{aligned}
& \operatorname{Inv}\left(T_{0}, p_{i}(i \in I) \mid p_{i}^{-1} c p_{i}=\phi_{i}^{*}(c) \text { for every } c \in C_{i},\right. \\
& \left.\qquad p_{i}^{-1} p_{i}=f_{i}, p_{i} p_{i}^{-1}=e_{i} \text { for every } i \in I\right) .
\end{aligned}
$$


Then $T$ is a locally full HNN extension of $T_{0}$. We show that $T$ is isomorphic to $S^{*}$. We define a mapping $\Phi$ of $S \cup\left\{t_{i} \mid i \in I\right\}$ into $T$ by $\Phi(s)=s$ for $s \in S$ and $\Phi\left(t_{i}\right)=p_{i}$ for each $i \in I$. Since the defining relations of $S^{*}$ are satisfied by $T$ under $\Phi$, the mapping $\Phi$ can be extended to a homomorphism by Proposition 1.1. We next define a mapping $\Psi$ of $T_{0} \cup\left\{p_{i} \mid i \in I\right\}$ into $S^{*}$ by $\Psi(s)=s$ for $s \in S, \Psi(e)=e$ for $e \in E\left(S^{*}\right)$ and $\Psi\left(p_{i}\right)=t_{i}$ for each $i \in I$. It is easy to check that the defining relations are satisfied by $S^{*}$ under $\Psi$. Hence, $\Psi$ can be extended to a homomorphism by Proposition 1.1. Apparently $\Psi \circ \Phi=i d_{S^{*}}$ and so $\Phi$ is injective. We next show that $\Phi$ is surjective. To prove this we show that every $e$ in $E\left(S^{*}\right)$ can be written as a product of $p_{i}$ 's and elements of $S$ in $T$. Take any $e \in E\left(S^{*}\right)$. Since $e$ is in $S^{*}$, the element $e$ can be written as a product of several stable letters $t_{i}$ 's and their inveNses and elements of $S$. Since $t_{i} t_{i}^{-1}, t_{i}^{-1} t_{i} \in E(S)$, we may assume that $e$ is written as

$$
e=s_{0} t_{i_{1}}^{\epsilon_{1}} s_{1} t_{i_{2}}^{\epsilon_{2}} s_{2} \cdots s_{n-1} t_{i_{n}}^{\epsilon_{n}} s_{n}
$$

where $s_{i} \in S$ for $i=0,1,2, \ldots, n$ and $\epsilon_{i}= \pm 1$ for $i=1,2, \ldots, n$. Note that

$$
\begin{aligned}
e & =e^{2}=e^{-1} e \\
& =s_{n}^{-1} t_{i_{n}}^{-\epsilon_{n}} s_{n-1}^{-1} t_{i_{n-1}}^{-\epsilon_{n-1}} s_{n-2}^{-1} \cdots s_{1}^{-1} t_{i_{1}}^{-\epsilon_{1}} s_{0}^{-1} s_{0} t_{i_{1}}^{\epsilon_{1}} s_{1} t_{i_{2}}^{\epsilon_{2}} s_{2} \cdots s_{n-1} t_{i_{n}}^{\epsilon_{n}} s_{n} .
\end{aligned}
$$

Therefore, every element of $e$ in $E\left(S^{*}\right)$ can be written as

$$
s_{n}^{-1} t_{i_{n}}^{-\epsilon_{n}} s_{n-1}^{-1} t_{i_{n-1}}^{-\epsilon_{n-1}} s_{n-2}^{-1} \cdots s_{1}^{-1} t_{i_{1}}^{-\epsilon_{1}} s_{0}^{-1} s_{0} t_{i_{1}}^{\epsilon_{1}} s_{1} t_{i_{2}}^{\epsilon_{2}} s_{2} \cdots s_{n-1} t_{i_{n}}^{\epsilon_{n}} s_{n},
$$

where $s_{i} \in S$ for $i=0,1,2, \ldots, n$ and $\epsilon_{i}= \pm 1$ for $i=1,2, \ldots, n$. We claim that if $e$ can be written in $S^{*}$ as above then we have

$$
e=s_{n}^{-1} p_{i_{n}}^{-\epsilon_{n}} s_{n-1}^{-1} p_{i_{n-1}}^{-\epsilon_{n-1}} s_{n-2}^{-1} \cdots s_{1}^{-1} p_{i_{1}}^{-\epsilon_{1}} s_{0}^{-1} s_{0} p_{i_{1}}^{\epsilon_{1}} s_{1} p_{i_{2}}^{\epsilon_{2}} s_{2} \cdots s_{n-1} p_{i_{n}}^{\epsilon_{n}} s_{n}
$$

in $T$ by induction on $n$. If $n=0$, then the claim is trivial. Suppose that the claim is true for non-negative integers less that $n(n \geq 1)$. Assume that

$$
e=s_{n}^{-1} t_{i_{n}}^{-\epsilon_{n}} s_{n-1}^{-1} t_{i_{n-1}}^{-\epsilon_{n-1}} s_{n-2}^{-1} \cdots s_{1}^{-1} t_{i_{1}}^{-\epsilon_{1}} s_{0}^{-1} s_{0} t_{i_{1}}^{\epsilon_{1}} s_{1} t_{i_{2}}^{\epsilon_{2}} s_{2} \cdots s_{n-1} t_{i_{n}}^{\epsilon_{n}} s_{n}
$$

in $S^{*}$. Let

$$
f=s_{n-1}^{-1} t_{i_{n-1}}^{-\epsilon_{n-1}} s_{n-2}^{-1} \cdots s_{1}^{-1} t_{i_{1}}^{-\epsilon_{1}} s_{0}^{-1} s_{0} t_{i_{1}}^{\epsilon_{1}} s_{1} t_{i_{2}}^{\epsilon_{2}} s_{2} \cdots t_{i_{n-1}}^{\epsilon_{n-1}} s_{n-1} .
$$

By the inductive hypothesis,

$$
f=s_{n-1}^{-1} p_{i_{n-1}}^{-\epsilon_{n-1}} s_{n-2}^{-1} \cdots s_{1}^{-1} p_{i_{1}}^{-\epsilon_{1}} s_{0}^{-1} s_{0} p_{i_{1}}^{\epsilon_{1}} s_{1} p_{i_{2}}^{\epsilon_{2}} s_{2} \cdots p_{i_{n-1}}^{\epsilon_{n-1}} s_{n-1}
$$

in $T$. Then we have

$$
e=s_{n}^{-1} t_{i_{n}}^{-\epsilon_{n}} f t_{i_{n}}^{\epsilon_{n}} s_{n}=s_{n}^{-1} t_{i_{n}}^{-\epsilon_{n}} f t_{i_{n}}^{\epsilon_{n}} t_{i_{n}}^{-\epsilon_{n}} t_{i_{n}}^{\epsilon_{n}} s_{n}
$$


Note that $f t_{i_{n}}^{\epsilon_{n}} t_{i_{n}}^{-\epsilon_{n}} \in E\left(S^{*}\right) t_{i_{n}}^{\epsilon_{n}} t_{i_{n}}^{-\epsilon_{n}}$. Then we have

$$
\begin{array}{rlrl}
t_{i_{n}}^{-\epsilon_{n}}\left(f t_{i_{n}}^{\epsilon_{n}} t_{i_{n}}^{-\epsilon_{n}}\right) t_{i_{n}}^{\epsilon_{n}} & =\left(\phi_{i}^{*}\right)^{\epsilon_{n}}\left(f t_{i_{n}}^{\epsilon_{n}} t_{i_{n}}^{-\epsilon_{n}}\right) & & \text { (by the definition of } \left.\phi_{i}^{*}\right) \\
& =p_{i_{n}}^{-\epsilon_{n}}\left(f t_{i_{n}}^{\epsilon_{n}} t_{i_{n}}^{-\epsilon_{n}}\right) p_{i_{n}}^{\epsilon_{n}} & & \text { (by the relation } p_{i}^{-1} c p_{i}=\phi_{i}^{*}(c) \\
& =p_{i_{n}}^{-\epsilon_{n}} f p_{i_{n}}^{\epsilon_{n}} p_{i_{n}}^{-\epsilon_{n}} p_{i_{n}}^{\epsilon_{n}} & & \text { for } \left.c \in C_{i} \in T\right) \\
& & \left(\text { as } t_{i_{i}} t_{i_{n}}^{-1}=e_{i_{n}}=p_{i_{n}} p_{i_{n}}^{-1}\right. \text { and } \\
& \left.t_{i_{n}}^{-1} t_{i_{n}}=f_{i_{n}}=p_{i_{n}}^{-1} p_{i_{n}} \in T\right)
\end{array}
$$

Therefore, we have

$$
\begin{aligned}
e & =s_{n}^{-1} t_{i_{n}}^{-\epsilon_{n}} f t_{i_{n}}^{\epsilon_{n}} t_{i_{n}}^{-\epsilon_{n}} t_{i_{n}}^{\epsilon_{n}} s_{n}=s_{n}^{-1} p_{i_{n}}^{-\epsilon_{n}} f p_{i_{n}}^{\epsilon_{n}} s_{n} \\
& =s_{n}^{-1} p_{i_{n}}^{-\epsilon_{n}} s_{n-1}^{-1} p_{i_{n-1}}^{-\epsilon_{n-1}} s_{n-2}^{-1} \cdots s_{1}^{-1} p_{i_{1}}^{-\epsilon_{1}} s_{0}^{-1} s_{0} p_{i_{1}}^{\epsilon_{1}} s_{1} p_{i_{2}}^{\epsilon_{2}} s_{2} \cdots p_{i_{n-1}}^{\epsilon_{n-1}} s_{n-1} p_{i_{n}}^{\epsilon_{n}} s_{n} .
\end{aligned}
$$

Hence, the claim is true for every non-negative integer. It follows that every $e \in E\left(S^{*}\right)$ can be written as a product of $p_{i}$ 's and elements of $S$. Since $T$ is generated by $S, E\left(S^{*}\right)$ and $p_{i}$ 's, the homomorphism $\Phi$ is surjective. Consequently $\Phi$ is an isomorphism of $S^{*}$ onto $T$.

\section{Normal form theorem}

Any element of an HNN extension of a group has a unique expression called a normal form (or canonical form). The method using such an expression is useful to study algorithmic and structural problems. In this section we obtain a normal form of an element of a locally full $\mathrm{HNN}$ extension of an inverse semigroup.

2.1. Normal form Let $S$ be an inverse semigroup, and let $H$ be an inverse subsemigroup of $S$. We define a relation $\sim_{H}$ on $S$.

For $x, y \in S$, we define

$$
x \sim_{H} y
$$

if one of the followings holds:

(E1) $x=y$.

(E2) $x^{-1} x, y^{-1} y \in E(H)$ and there exists $h \in H$ such that $x h=y$ with $x^{-1} x=h h^{-1}$.

PROPOSITION 2.1. The relation $\sim_{H}$ is an equivalence relation on $S$.

Proof. Clearly, $x \sim_{H} x$ for every $x \in S$. Suppose that $x \sim_{H} y$. Then either (E1) or (E2) holds. In the case of (E1), $x=y$ and so clearly $y \sim_{H} x$. We assume (E2). 
Then, we have $x^{-1} x, y^{-1} y \in E(H)$ and $x h=y$ and $x^{-1} x=h h^{-1}$ for some $h \in H$. Then, $y h^{-1}=x h h^{-1}=x x^{-1} x=x$. We note that $h^{-1} \in H$. It is easy to see that $y^{-1} y=h^{-1}\left(h^{-1}\right)^{-1}$. Hence, $y \sim_{H} x$.

Suppose now that $x \sim_{H} y, y \sim_{H} z$. We consider only the case that $x^{-1} x, y^{-1} y$, $z^{-1} z \in E(H)$ and $x h=y, y k=z, x^{-1} x=h h^{-1}$ and $y^{-1} y=k k^{-1}$ for some $h, k$ in $H$. Then $z=x(h k)$ and $h k \in H$. It is easy to see that $x^{-1} x=(h k)(h k)^{-1}$. Hence, $x \sim_{H} z$. Consequently, we have shown that $\sim_{H}$ is an equivalence.

The $\sim_{H}$ class of $S$ containing an element $x$ is denoted by $\bar{x}$. We denote the equivalence class of $S$ containing $x$ for Green's relation $\mathscr{H}, \mathscr{R}, \mathscr{L}$ and $\mathscr{D}$ by $H_{S}(x)$, $R_{S}(x), L_{S}(x)$ and $D_{S}(x)$, respectively.

LEMMA 2.2. For an element $h$ of $H$ we have $\bar{h}=R_{S}(h) \cap H=R_{H}(h)$.

PRoof. Suppose $x \in \bar{h}$. Then $x \sim_{H} h$, and hence, $x=h$ or $h k=x$ and $h^{-1} h=k k^{-1}$ for some $k \in H$. Since $h, k \in H$, we have $x=h k \in H$. Then $x \mathscr{R} h$ in $S$, since $x=h k$ and $h=x k^{-1}$. Hence, $x \in R_{S}(h) \cap H$. Take $x$ from $R_{S}(h) \cap H$. Then $x \mathscr{R} h$ in $S$ and $x \in H$. Hence, $x x^{-1}=h h^{-1}$ and so $x \in R_{H}(h)$. Take $x$ from $R_{H}(h)$. Then, $x \in H$ and $x \mathscr{R} h$ in $H$ and so $x x^{-1}=h h^{-1}$. Then, we have $h\left(h^{-1} x\right)=x x^{-1} x=x$. We note that $h^{-1} x \in H$ (as $\left.h, x \in H\right)$. It is easy to see that $\left(h^{-1} x\right)\left(h^{-1} x\right)^{-1}=h^{-1} h$. Hence, $x \in \bar{h}$.

LEMMA 2.3. For $x, y \in S, x \sim_{H} y$ if and only if

(1) $x=y$ and $x^{-1} x \notin E(H)$ or

(2) $x x^{-1}=y y^{-1}, x^{-1} y \in H$ and $x^{-1} x, y^{-1} y \in E(H)$.

PROOF. 'Only if' part: Suppose $x \sim_{H} y$. If (E1) holds, then we have (1) or (2) according to whether or not $x^{-1} x$ is in $E(H)$. We now suppose that (E2) holds. Then $x^{-1} x, y^{-1} y \in E(H)$. There exists $h \in H$ such that $x h=y$ and $x^{-1} x=h h^{-1}$. Then we have $x^{-1} y=x^{-1} x h \in E(H) H \subset H$. It is easy to see that $y y^{-1}=x x^{-1}$. Hence, (2) holds.

'If' part: Suppose (2) is true. Then $x^{-1} x, y^{-1} y \in E(H)$. Set $x^{-1} y=h \in H$. Then we have $x h=x x^{-1} y=y y^{-1} y=y$. It is easy to see that $x^{-1} x=h h^{-1}$. Hence, $x \sim_{H} y$. If (1) holds, clearly $x \sim_{H} y$.

PROPOSITION 2.4. If $H=E(S)$, then $\sim_{H}$ is the identity relation on $S$.

PRoOF. Suppose $x \sim_{H} y$ and the condition (E2) holds. Then there exists $h \in E(S)$ such that $x h=y$ and $x^{-1} x=h h^{-1}$. Since $h \in E(S)$, we have $x^{-1} x=h h^{-1}=h$. Then $y=x h=x x^{-1} x=x$. Hence, $x=y$ and so $\sim_{H}$ is the identity on $S$. 
PROPOSITION 2.5. If $H=S$, then $\sim_{H}$ is identical to Green's $\mathscr{R}$-relation.

PROOF. Obviously $\sim_{H}$ is included in $\mathscr{R}$. Suppose $x \mathscr{R} y$. Then, $x x^{-1}=y y^{-1}$ and so $x=x x^{-1} x=y y^{-1} x$. It is easy to see that $y^{-1} y=\left(y^{-1} x\right)\left(y^{-1} x\right)^{-1}$. Note that $y^{-1} x \in S=H$. Hence, (E2) holds, and so, $x \sim_{H} y$. It follows that $\sim_{H}$ is identical with $\mathscr{R}$.

We begin to seek a unique expression of an element of a locally full HNN extension. Let $S$ be an inverse semigroup, $A_{i}$ and $B_{i}(i \in I)$ inverse subsemigroups of $S$. Suppose that $e_{i} \in A_{i} \subset e_{i} S e_{i}, f_{i} \in B_{i} \subset f_{i} S f_{i}$ for some idempotents $e_{i}, f_{i}$ of $S$ and that $\phi_{i}: A_{i} \rightarrow B_{i}$ is an isomorphism for every $i \in I$. We also assume that $E\left(A_{i}\right)=E\left(e_{i} S e_{i}\right)$ and $E\left(B_{i}\right)=E\left(f_{i} S f_{i}\right)$ for every $i \in I$. Let $S^{*}$ be the inverse semigroup presented by

$$
\begin{aligned}
& \operatorname{Inv}\left(S, t_{i}(i \in I) \mid t_{i}^{-1} a t_{i}=\phi_{i}(a) \text { for every } a \in A_{i},\right. \\
& \left.\qquad t_{i}^{-1} t_{i}=f_{i}, t_{i} t_{i}^{-1}=e_{i} \text { for every } i \in I\right) .
\end{aligned}
$$

Note that $S^{*}$ is the locally full HNN extension of $S$ associated with the isomorphisms $\phi_{i}(i \in I)$. In the rest of the paper we assume that $S, A_{i}, B_{i}$ and $S^{*}$ are defined as above.

We choose and fix a set $\mathrm{C}\left(\sim_{A_{i}}\right)$ of $\sim_{A_{i}}$ representatives of $S$ for each $i \in I$. Similarly, we choose and fix a set $\mathrm{C}\left(\sim_{B_{i}}\right)$ of $\sim_{B_{j}}$ representatives of $S$ for each $j \in I$. If $r \in \mathrm{C}\left(\sim_{A_{i}}\right)$ is in the $\sim_{A_{i}}$ class $R_{S}(e) \cap A_{i}$ (this is a $\sim_{A_{i}}$ class by Lemma 2.2) for some idempotent $e \in E\left(A_{i}\right)$, then we assume $r=e$. Similarly, if $s \in \mathrm{C}\left(\sim_{B_{i}}\right)$ is in the $\sim_{B_{j}}$ class $R_{S}(f) \cap B_{j}$ for some idempotent $f \in E\left(B_{j}\right)$, then we assume $s=f$.

Let $X$ be a sequence

$$
\left(x_{0}, t_{i_{1}}^{\epsilon_{1}}, x_{1}, t_{i_{2}}^{\epsilon_{2}}, \ldots, t_{i_{n}}^{\epsilon_{n}}, x_{n}\right)
$$

where $n \geq 0, x_{k} \in S$ for every $k=0,1,2, \ldots, n, t_{i_{l}}$ is a stable letter and $\epsilon_{l}= \pm 1$ for every $l=1,2, \ldots, n$. We consider the following conditions on the sequence $X$ :

(C1) If $\epsilon_{k}=1$, then $x_{k-1}^{-1} x_{k}>\in E\left(A_{i_{k}}\right)$. If $\epsilon_{k}=-1$, then $x_{k-1}^{-1} x_{k-1} \in E\left(B_{i_{k}}\right)$, and $\phi_{i_{k}}^{\epsilon_{k}}\left(x_{k-1}^{-1} x_{k-1}\right)=x_{k} x_{k}^{-1}$ for every $k=1,2, \ldots, n$.

(C2) If $\epsilon_{k}=1$, then $x_{k-1}$ belongs to $\mathrm{C}\left(\sim_{A_{i_{k}}}\right)$, and if $\epsilon_{k}=-1$, then $x_{k-1}$ belongs to $\mathrm{C}\left(\sim_{B_{i_{k}}}\right)$ for every $k=1,2, \cdots n$.

(C3) There exists no subsequence of the form $\left(t_{i}^{-1}, e, t_{i}\right)\left(e \in E\left(A_{i}\right)\right)$ nor $\left(t_{i}, e, t_{i}^{-1}\right)$ $\left(e \in E\left(B_{i}\right)\right)$.

(C4) There exists no subsequence of the form $\left(t_{i}^{-1}, a, t_{i}\right)\left(a \in A_{i}\right)$ nor $\left(t_{i}, b, t_{i}^{-1}\right)$ $\left(b \in B_{i}\right)$.

A sequence satisfying $(\mathrm{C} 1),(\mathrm{C} 2)$ and $(\mathrm{C} 3)((\mathrm{C} 1)$ and $(\mathrm{C} 4))$ is called normal (reduced). Note that a normal sequence is always reduced. A pinch is a word of the form $t_{i}^{-1} a t_{i}$ 
( $\left.a \in A_{i}\right)$ or $t_{i} b t_{i}^{-1}\left(b \in B_{i}\right)$, where $t_{i}$ is a stable letter. Let $x$ be an element of $S^{*}$. Suppose that $x$ is written as

$$
x_{0} t_{i_{1}}^{\epsilon_{1}} x_{1} t_{i_{2}}^{\epsilon_{2}} \cdots t_{i_{n}}^{\epsilon_{n}} x_{n}
$$

If the sequence

$$
\left(x_{0}, t_{i_{1}}^{\epsilon_{1}}, x_{1}, t_{i_{2}}^{\epsilon_{2}}, \cdots, t_{i_{n}}^{\epsilon_{n}}, x_{n}\right)
$$

satisfies the condition ( $\mathrm{Cl}$ ), then we say that $x$ has a form (2.3). Moreover, if the sequence (2.4) is normal (reduced), then we say that $x$ has a normal form (reduced form) (2.3). Note that a form is reduced if and only if it contains no pinch. We remark that a form in an inverse semigroup corresponds to the trace product in the corresponding inductive groupoid. The inductive groupoid approach is employed in [4].

LEMMA 2.6. If an element $x$ in $S^{*}$ has a form $x_{0} t_{i_{1}}^{\epsilon_{1}} x_{1} t_{i_{2}}^{\epsilon_{2}} \cdots t_{i_{n}}^{\epsilon_{n}} x_{n}$ then the following hold:

(1) $x x^{-1}=x_{0} x_{0}^{-1}$.

(2) $x^{-1} x=x_{n}^{-1} x_{n}$

PROOF. We prove (1) using induction on $n$. If $n=0$, then $x=x_{0}$ and the claim is trivially true. Next suppose that the claim is true for every non-negative integer less than $n$ and that $x$ has the form $x_{0} t_{i_{1}}^{\epsilon_{1}} x_{1} t_{i_{2}}^{\epsilon_{2}} \cdots t_{i_{n}}^{\epsilon_{n}} x_{n}$. By the inductive hypothesis, we have

$$
\left(x_{0} t_{i_{1}}^{\epsilon_{1}} x_{1} t_{i_{2}}^{\epsilon_{2}} \cdots t_{i_{n-1}}^{\epsilon_{n-1}} x_{n-1}\right)\left(x_{0} t_{i_{1}}^{\epsilon_{1}} x_{1} t_{i_{2}}^{\epsilon_{2}} \cdots t_{i_{n-1}}^{\epsilon_{n-1}} x_{n-1}\right)^{-1}=x_{0} x_{0}^{-1}
$$

Therefore

$$
\begin{aligned}
x x^{-1}= & \left(x_{0} t_{i_{1}}^{\epsilon_{1}} x_{1} t_{i_{2}}^{\epsilon_{2}} \cdots t_{i_{n}}^{\epsilon_{n}} x_{n}\right)\left(x_{0} t_{i_{1}}^{\epsilon_{1}} x_{1} t_{i_{2}}^{\epsilon_{2}} \cdots t_{i_{n}}^{\epsilon_{n}} x_{n}\right)^{-1} \\
= & \left(x_{0} t_{i_{1}}^{\epsilon_{1}} x_{1} t_{i_{2}}^{\epsilon_{2}} \cdots t_{i_{n-1}}^{\epsilon_{n-1}} x_{n-1}\right) t_{i_{n}}^{\epsilon_{n}} x_{n} x_{n}^{-1} t_{i_{n}}^{\epsilon_{n}}\left(x_{0} t_{i_{1}}^{\epsilon_{1}} x_{1} t_{i_{2}}^{\epsilon_{2}} \cdots t_{i_{n-1}}^{\epsilon_{n-1}} x_{n-1}\right)^{-1} \\
= & \left(x_{0} t_{i_{1}}^{\epsilon_{1}} x_{1} t_{i_{2}}^{\epsilon_{2}} \cdots t_{i_{n-1}}^{\epsilon_{n-1}} x_{n-1}\right) x_{n-1}^{-1} x_{n-1}\left(x_{0} t_{i_{1}}^{\epsilon_{1}} x_{1} t_{i_{2}}^{\epsilon_{2}} \cdots t_{i_{n-1}}^{\epsilon_{n-1}} x_{n-1}\right)^{-1} \\
& \text { (by (C1) and the relations } t_{i}^{-1} a t_{i}=\phi_{i}(a) \text { for all } a \in A_{i} \\
& \text { and } \left.t_{i} b t_{i}^{-1}=\phi_{i}^{-1}(b) \text { for all } b \in B_{i}\right) \\
= & \left(x_{0} t_{i_{1}}^{\epsilon_{1}} x_{1} t_{i_{2}}^{\epsilon_{2}} \cdots t_{i_{n-1}}^{\epsilon_{n-1}} x_{n-1}\right)\left(x_{0} t_{i_{1}}^{\epsilon_{1}} x_{1} t_{i_{2}}^{\epsilon_{2}} \cdots t_{i_{n-1}}^{\epsilon_{n-1}} x_{n-1}\right)^{-1}=x_{0} x_{0}^{-1} .
\end{aligned}
$$

This completes the induction. Similarly we can prove (2).

2.2. Normal form theorem In this section we prove the normal form theorem for a locally full HNN extension.

THEOREM 2.7. Let $S$ be an inverse semigroup, and let $A_{i}$ and $B_{i}$ be inverse subsemigroups such that $e_{i} \in A_{i} \subset e_{i} S e_{i}, E\left(A_{i}\right)=E\left(e_{i} S e_{i}\right), f_{i} \in B_{i} \subset f_{i} S f_{i}$ and 
$E\left(B_{i}\right)=E\left(f_{i} S f_{i}\right)$ for every $i \in I$. Let $\phi_{i}: A_{i} \rightarrow B_{i}$ be an isomorphism of $A_{i}$ onto $B_{i}$ for every $i \in 1$. Let $S^{*}$ be the locally full $\mathrm{HNN}$ extension presented by (2.2). Every element of $S^{*}$ has a unique normal form (2.3).

The proof is divided into four lemmas. Lemma 2.8 shows that every element has a form and also provides an algorithm that finds a form. Lemma 2.9 shows that we can construct a reduced form from a form and provides an algorithm for doing so. Lemma 2.10 shows that we can obtain a normal form from a reduced form. Lemma 2.11 guarantees the uniqueness of the normal form.

LEMMA 2.8. Every element of $S^{*}$ has a form. Moreover, if the isomorphisms $\phi_{i}$ and $\phi_{i}^{-1}$ are effectively computable for every $i \in I$, then we can effectively find a form for any element of $S^{*}$.

PROOF. Take an element $x \in S^{*}$. Note that $S^{*}$ is generated by $S$ and the stable letters $t_{i}$. Since $t_{i} t_{i}^{-1}=e_{i}$ and $t_{i}^{-1} t_{i}=f_{i}$, the element $x$ can be written as

$$
x=y_{0} t_{i_{1}}^{\delta_{1}} y_{1} t_{i_{2}}^{\delta_{2}} \cdots t_{i_{m}}^{\delta_{m}} y_{m},
$$

where $y_{j} \in S$ for $j=0,1, \ldots, m$ and $\delta_{j}= \pm 1$ for $j=1,2, \ldots, m$. Since we have $t_{i} t_{i}^{-1}=e_{i}$ and $t_{i}^{-1} t_{i}=f_{i}$, we may assume that $y_{0}^{-1} y_{0} \leq t_{i_{1}}^{\delta_{1}} t_{i_{1}}^{-\delta_{1}}, y_{m} y_{m}^{-1} \leq t_{i_{m}}^{-\delta_{m}} t_{i_{m}}^{\delta_{m}}$, $y_{k} y_{k}^{-1} \leq t_{i_{k}}^{-\delta_{k}} t_{i_{k}}^{\delta_{k}}$ for $k=1,2, \ldots, m$, and $y_{k}^{-1} y_{k} \leq t_{i_{k+1}}^{\delta_{k+1}} t_{i_{k+1}}^{-\delta_{k+1}}$ for $k=0,1, \ldots, m-1$. Then

$$
y_{k-1}^{-1} y_{k-1} \in E\left(A_{i_{k}}\right) \quad \text { if } \delta_{k}=1
$$

and

$$
y_{k-1}^{-1} y_{k-1} \in E\left(B_{i_{k}}\right) \quad \text { if } \delta_{k}=-1
$$

for $k=1,2, \ldots, m$. Using induction on $m$, we show that if $x$ is written as $y_{0} t_{i_{1}}^{\delta_{1}} y_{1} t_{i_{2}}^{\delta_{2}} \cdots t_{i_{m}}^{\delta_{m}} y_{m}$ satisfying (2.5) and (2.6), then the element $x$ has a form $x_{0} t_{k_{1}}^{\delta_{1}} x_{1} t_{k_{2}}^{\delta_{2}} \cdots$ $t_{k_{m}}^{\delta_{m}} x_{m}$. If $m=0$, the result is trivially true. Suppose that the result is true for any non-negative integer less thap $m(1 \leq m)$, and that $x$ is written as $y_{0} t_{i_{1}}^{\delta_{1}} y_{1} t_{i_{2}}^{\delta_{2}} \cdots t_{i_{m}}^{\delta_{m}} y_{m}$ satisfying (2.5) and (2.6). We set

$$
z=y_{0} t_{i_{1}}^{\delta_{1}} y_{1} t_{i_{2}}^{\delta_{2}} \cdots y_{m-2} t_{i_{m-1}}^{\delta_{m-1}} y_{m-1} \phi_{i_{m}}^{-\delta_{m}}\left(y_{m} y_{m}^{-1}\right) \text {. }
$$

Note that $x=z t^{\delta_{m}} y_{m}$ since $\phi^{-\delta_{m}}\left(y_{m} y_{m}^{-1}\right) t^{\delta_{m}}=t^{\delta_{m}} y_{m} y_{m}^{-1}$. By inductive hypothesis, the element $z$ has a form $z_{0} t_{i_{1}}^{\delta_{1}} z_{1} t_{i_{2}}^{\delta_{2}} \cdots z_{m-2} t_{i_{m-1}}^{\delta_{m-1}} z_{m-1}$. It is easily verified that

$$
z_{0} t_{i_{1}}^{\delta_{1}} z_{1} t_{i_{2}}^{\delta_{2}} \cdots z_{m-2} t_{i_{m-1}}^{\delta_{m-1}} z_{m-1} t_{i_{m}}^{\delta_{m}} \phi^{\delta_{m}}\left(z_{m-1}^{-1} z_{m-1}\right) y_{m}
$$

is a form for $x$. It is also easy to see that there exists an algorithm that finds a form for an element if the isomorphisms $\phi_{i}$ and $\phi_{i}^{-1}(i \in I)$ are effectively computable. 
LEMMA 2.9. Every element of $S^{*}$ has a reduced form. Moreover, if the isomorphisms $\phi_{i}$ and $\phi_{i}^{-1}$ are effectively computable and the membership problems for $A_{i}$ and $B_{i}$ in $S$ are solvable for every $i \in I$, then we can effectively find a reduced form for a given element $x$ of $S^{*}$ provided that a form for $x$ is given.

PROOF. Take $x \in S^{*}$. Suppose that $x$ has a form $x=x_{0} t_{i_{1}}^{\delta_{1}} x_{1} t_{i_{2}}^{\delta_{2}} \cdots t_{i_{n}}^{\delta_{n}} x_{n}$. If the form contains a pinch, that is, a subword of the form $t_{i_{k}}^{\delta_{k}} x_{k} t_{i_{k+1}}^{\delta_{k+1}}$, where $i_{k}=i_{k+1}, x_{k} \in A_{i_{k}}$, $\delta_{k}=-1$ and $\delta_{k+1}=1$ (or $i_{k}=i_{k+1}, x_{k} \in B_{i_{k}}, \delta_{k}=1$ and $\delta_{k+1}=-1$ ), then we replace it by $\phi_{i_{k}}^{-\delta_{k}}\left(x_{k}\right)$. Recall that $\phi_{i_{k}}^{-\delta_{k}}\left(x_{k}\right)=t_{i_{k}}^{\delta_{k}} x_{k} t_{i_{k+1}}^{\delta_{k+1}}$ in $S^{*}$. Then we have

$$
x=x_{0} t_{i_{1}}^{\delta_{1}} x_{1} t_{i_{2}}^{\delta_{2}} \cdots t_{i_{k-1}}^{\delta_{k-1}} x_{k-1} \phi_{i_{k}}^{-\delta_{k}}\left(x_{k}\right) x_{k+1} t_{i_{k+2}}^{\delta_{k+2}} \cdots t_{i_{n}}^{\delta_{n}} x_{n}
$$

It is easy to see that this is a form of $x$. We continue this process until all pinches disappear, and then the resulting form is reduced. Obviously we can construct an algorithm to find a reduce form using the argument above if the isomorphisms $\phi_{i}$ and $\phi_{i}^{-1}(i \in I)$ are effectively computable and the membership problems for $A_{i}$ and $B_{i}$ in $S$ are solvable for every $i \in I$.

LEMMA 2.10. Every element of $S^{*}$ has a normal form. Furthermore, if $x$ has a reduced form $x_{0} t_{i_{1}}^{\epsilon_{1}} x_{1} t_{i_{2}}^{\epsilon_{2}} \cdots t_{i_{n}}^{\epsilon_{n}} x_{n}$, then a normal form for $x$ is written as $u_{0} t_{i_{1}}^{\epsilon_{1}} u_{1} t_{i_{2}}^{\epsilon_{2}} \cdots t_{i_{n}}^{\epsilon_{n}} u_{n}$ for some $u_{0}, u_{1}, \ldots u_{n} \in S$.

PROOF. Take an element $x$ of $S^{*}$. By Lemma 2.8 and Lemma 2.9, $x$ has a reduced form $x_{0} t_{i_{1}}^{\epsilon_{1}} x_{1} t_{i_{2}}^{\epsilon_{2}} \cdots t_{i_{n}}^{\epsilon_{n}} x_{n}$. If $\epsilon_{1}=1$, then we rewrite $x_{0}$ as $x_{0}=u_{0} c_{0}$, where $u_{0}$ is a $\sim_{A_{i_{1}}}$ representative for $x_{0}$ and $c_{0} \in A_{i_{1}}$ with $u_{0}^{-1} u_{0}=c_{0} c_{0}^{-1}$. If $\epsilon_{1}=-1$, then we rewrite $x_{0}$ as $x_{0}=u_{0} c_{0}$, where $u_{0}$ is a $\sim_{B_{i_{1}}}$ representative for $x_{0}$ and $c_{0} \in B_{i_{1}}$ with $u_{0}^{-1} u_{0}=c_{0} c_{0}^{-1}$. Then we have

$$
x=u_{0} c_{0} t_{i_{1}}^{\epsilon_{1}} x_{1} t_{i_{2}}^{\epsilon_{2}} \cdots t_{i_{n}}^{\epsilon_{n}} x_{n}=u_{0} t_{i_{1}}^{\epsilon_{1}} \phi_{i_{1}}^{\epsilon_{1}}\left(c_{0}\right) x_{1} t_{i_{2}}^{\epsilon_{2}} \cdots t_{i_{n}}^{\epsilon_{n}} x_{n} .
$$

It is easy to see that the resulting sequence is a reduced form for $x$. Then we have $\phi^{\epsilon_{1}}\left(c_{0}\right) x_{1}=u_{1} c_{1}$ where $c_{1}$ is a $\sim_{A_{i_{2}}}$ or $\sim_{B_{i_{2}}}$ representative according to $\epsilon_{2}= \pm 1$. We rewrite the form by applying a similar argument to the subword $\phi_{i_{1}}^{\epsilon_{1}}\left(c_{0}\right) x_{1} t_{i_{2}}^{\epsilon_{2}}$ and continue the same process. We eventually get a form $u_{0} t_{i_{1}}^{\epsilon_{1}} u_{1} t_{i_{2}}^{\epsilon_{2}} \cdots t_{i_{n}}^{\epsilon_{n}} u_{n}$ for $x$. Clearly it is a normal form for $x$.

LEMMA 2.11. A normal form for any element of $S^{*}$ is unique.

PRoOF. We use the Artin-van der Waerden method. Let $\mathrm{N}$ be the set of normal sequences. Let $\operatorname{Sym}(N)$ be the symmetric inverse semigroup on $N$. We assume that an element of $\operatorname{Sym}(N)$ acts from the right. We define a mapping $\Phi$ of $S \cup\left\{t_{i}, t_{i}^{-1} \mid i \in I\right\}$ 
into $\operatorname{Sym}(\mathrm{N})$ as follows. For any $X \in \mathrm{N}$, if $X=\left(x_{0}, t_{i_{1}}^{\epsilon_{1}}, x_{1}, t_{i_{2}}^{\epsilon_{2}}, \ldots, t_{i_{n}}^{\epsilon_{n}}, x_{n}\right)$, then we define $\mathbf{z}(X)$ to be the last component $x_{n}$. For $s \in S$, we define $\Phi(s)$. We set

$$
\operatorname{Dom}(\Phi(s))=\left\{X \in \mathbf{N} \mid \mathbf{z}(X) \in S s s^{-1}\right\}
$$

and for $X=\left(x_{0}, t_{i_{1}}^{\epsilon_{1}}, \ldots, t_{i_{n}}^{\epsilon_{n}}, x_{n}\right) \in \operatorname{Dom}(\Phi(s))$

$$
X \Phi(s)=\left(x_{0}, t_{i_{1}}^{\epsilon_{1}}, \ldots, t_{i_{n}}^{\epsilon_{n}}, x_{n} s\right) .
$$

Clearly $X \Phi(s)$ satisfies the condition (C1), (C2) and (C3), and hence, it belongs to $N$. It is easy to see that $\operatorname{Ran}(\Phi(s))=\left\{X \in \mathrm{N} \mid \mathbf{z}(X) \in S s^{-1} s\right\}$ and the inverse mapping of $\Phi(s)$ is $\Phi\left(s^{-1}\right)$ for every $s \in S$. Therefore, $\Phi(s)$ belongs to $\operatorname{Sym}(\mathrm{N})$. It is also easy to see that for $s, r \in S$ we have

$$
\Phi(s r)=\Phi(s) \Phi(r)
$$

that is, $\Phi$ is a homomorphism on $S$.

We now define $\Phi\left(t_{i}\right)$ and $\Phi\left(t_{i}^{-1}\right)$ for every $i \in I$. We set

$$
\operatorname{Dom}\left(\Phi\left(t_{i}\right)\right)=\left\{X \in \mathbf{N} \mid \mathbf{z}(X) \in S e_{i}\right\}
$$

and

$$
\operatorname{Ran}\left(\Phi\left(t_{i}\right)\right)=\left\{X \in \mathbf{N} \mid \mathbf{z}(X) \in S f_{i}\right\},
$$

where $t_{i} t_{i}^{-1}=e_{i}$ and $t_{i}^{-1} t_{i}=f_{i}$. Note that if $X \in \operatorname{Dom}\left(\Phi\left(t_{i}\right)\right)$, then $\mathbf{z}(X)^{-1} \mathbf{z}(X) \in$ $E\left(A_{i}\right)$ since $E\left(A_{i}\right)=E\left(e_{i} S e_{i}\right)$. Take $X=\left(x_{0}, t_{i_{1}}^{\epsilon_{1}}, \ldots, t_{i_{n}}^{\epsilon_{n}}, x_{n}\right)$ from $\operatorname{Dom}\left(\Phi\left(t_{i}\right)\right)$. We define $\Phi\left(t_{i}\right)$ by

$$
\begin{array}{ll}
X \Phi\left(t_{i}\right)=\left(x_{0}, t_{i_{1}}^{\epsilon_{1}}, \ldots, t_{i_{n-1}}^{\epsilon_{n-1}}, x_{n-1} \phi_{i}\left(x_{n}\right)\right) \quad & \text { if } \mathbf{z}(X)=x_{n} \in A_{i_{n}}, \\
& i=i_{n} \text { and } \epsilon_{n}=-1,
\end{array}
$$

where $x_{n}=y_{n} a$ and $y_{n}$ is a $\sim_{A_{i}}$ representative, $y_{n}^{-1} y_{n}=a a^{-1}$ and $a \in A_{i}$. Similarly, we define $\phi\left(t_{i}^{-1}\right)$ as follows. We set

$$
\operatorname{Dom}\left(\Phi\left(t_{i}^{-1}\right)\right)=\left\{X \in \mathbf{N} \mid \mathbf{z}(X) \in S f_{i}\right\}
$$

and

$$
\operatorname{Ran}\left(\Phi\left(t_{i}^{-1}\right)\right)=\left\{X \in \mathrm{N} \mid \mathbf{z}(X) \in S e_{i}\right\} .
$$

Note that if $X \in \operatorname{Dom}\left(\Phi\left(t_{i}^{-1}\right)\right)$ then $\mathbf{z}(X)^{-1} \mathbf{z}(X) \in E\left(B_{i}\right)$ since $E\left(B_{i}\right)=E\left(f_{i} S f_{i}\right)$. Take $X=\left(x_{0}, t_{i_{1}}^{\epsilon_{1}}, \ldots, t_{i_{n}}^{\epsilon_{n}}, x_{n}\right)$ from $\operatorname{Dom}\left(\Phi\left(t_{i}^{-1}\right)\right)$. We define $\Phi\left(t_{i}^{-1}\right)$ by

$$
\begin{array}{ll}
X \Phi\left(t_{i}^{-1}\right)=\left(x_{0}, t_{i_{1}}^{\epsilon_{1}}, \ldots, t_{i_{n-1}}^{\epsilon_{n-1}}, x_{n-1} \phi_{i}^{-1}\left(x_{n}\right)\right), & \text { if } \mathbf{z}(X)=x_{n} \in B_{i_{n}}, \\
& i=i_{n} \text { and } \epsilon_{n}=1,
\end{array}
$$

$$
X \Phi\left(t_{i}^{-1}\right)=\left(x_{0}, t_{i_{1}}^{\epsilon_{1}}, \ldots, t_{i_{n-1}}^{\epsilon_{n-1}}, x_{n-1}, t_{i_{n}}^{\epsilon_{n}}, y_{n}, t_{i}^{-1}, \phi_{i}^{-1}(b)\right) \quad \text { otherwise }
$$


where $x_{n}=y_{n} b$ and $y_{n}$ is a $\sim_{B_{i}}$ representative, $y_{n}^{-1} y_{n}=b b^{-1}$ and $b \in B_{i}$.

If all defining relations for $S^{*}$ are preserved under $\Phi$ then the mapping $\Phi$ can be extended to a homomorphism $\Phi: S^{*} \rightarrow \operatorname{Sym}(\mathrm{N})$ by Proposition 1.1. To show all relations are preserved, we prove

$$
\begin{aligned}
\Phi\left(t_{i}\right) \Phi\left(t_{i}^{-1}\right) & =\Phi\left(e_{i}\right) & & \text { for every } i \in I, \\
\Phi\left(t_{i}^{-1}\right) \Phi\left(t_{i}\right) & =\Phi\left(f_{i}\right) & & \text { for every } i \in I, \\
\Phi\left(t_{i}^{-1}\right) \Phi(a) \Phi\left(t_{i}\right) & =\Phi\left(\phi_{i}(a)\right) & & \text { for every } a \in A_{i} .
\end{aligned}
$$

Since $\Phi$ is a homomorphism on $S$, it preserves the relations for $S$.

We prove the relation (2.13). Since $e_{i}$ is an idempotent, we have

$$
\operatorname{Dom}\left(\Phi\left(e_{i}\right)\right)=\left\{X \in \mathbf{N} \mid \mathbf{z}(X) \in S e_{i}\right\}
$$

by (2.7). Then we have

$$
X \Phi\left(e_{i}\right)=\left(x_{0}, t_{i_{1}}^{\epsilon_{1}}, \ldots, t_{i_{n}}^{\epsilon_{n}}, x_{n} e_{i}\right)=\left(x_{0}, t_{i_{1}}^{\epsilon_{1}}, \ldots, t_{i_{n}}^{\epsilon_{n}}, x_{n}\right)=X
$$

for any $X=\left(x_{0}, t_{i_{1}}^{\epsilon_{1}}, \ldots, t_{i_{n}}^{\epsilon_{n}}, x_{n}\right) \in \operatorname{Dom}\left(\Phi\left(e_{i}\right)\right)$ by (2.8). Therefore, $\Phi\left(e_{i}\right)$ is the identity mapping on $\operatorname{Dom}\left(\Phi\left(e_{i}\right)\right)$. On the other hand, we have

$$
\operatorname{Ran}\left(\Phi\left(t_{i}\right)\right)=\left\{X \in \mathbf{N} \mid \mathbf{z}(X) \in S f_{i}\right\}=\operatorname{Dom}\left(\Phi\left(t_{i}^{-1}\right)\right) .
$$

It follows that

$$
\begin{aligned}
\operatorname{Dom}\left(\Phi\left(t_{i}\right) \Phi\left(t_{i}^{-1}\right)\right) & =\left(\operatorname{Ran}\left(\Phi\left(t_{i}\right)\right) \cap \operatorname{Dom}\left(\Phi\left(t_{i}^{-1}\right)\right)\right) \Phi\left(t_{i}\right)^{-1} \\
& =\operatorname{Ran}\left(\Phi\left(t_{i}\right)\right) \Phi\left(t_{i}\right)^{-1}=\operatorname{Dom}\left(\Phi\left(t_{i}\right)\right) \\
& =\left\{X \in \mathbf{N} \mid \mathbf{z}(X) \in \operatorname{Se}_{i}\right\}=\operatorname{Dom}\left(\Phi\left(e_{i}\right)\right) .
\end{aligned}
$$

Take an element $X$ from $\operatorname{Dom}\left(\Phi\left(e_{i}\right)\right)=\left\{X \in N \mid \mathbf{z}(X) \in S e_{i}\right\}$. Suppose that $X=\left(x_{0}, t_{i_{1}}^{\epsilon_{1}}, \ldots, t_{i_{n}}^{\epsilon_{n}}, x_{n}\right)$, where $\mathbf{z}(X)=x_{n} \in S e_{i}$. We compute $X \Phi\left(t_{i}\right) \Phi\left(t_{i}^{-1}\right)$. In computing $X \Phi\left(t_{i}\right)$ for $X$ and $t_{i}$, there are two possible cases; (2.9) and (2.10).

Suppose that (2.9) is true. Then we have $x_{n} \in A_{i}, i=i_{n}$ and $\epsilon_{n}=-1$. Remark that in this case, it cannot happen that $x_{n-1} \in B_{i}, i=i_{n-1}$ and $\epsilon_{n-1}=1$ since $X \in \mathrm{N}$. We have

$$
X \Phi\left(t_{i}\right)=\left(x_{0}, t_{i_{1}}^{\epsilon_{1}}, \ldots, t_{i_{n-1}}^{\epsilon_{n-1}}, x_{n-1} \phi_{i}\left(x_{n}\right)\right) .
$$

By the remark above, $t_{i_{n-1}}^{\epsilon_{n-1}} x_{n-1} \phi_{i}\left(x_{n}\right) t_{i}^{-1}$ is not a pinch, and so, we have

$$
\begin{aligned}
X \Phi\left(t_{i}\right) \Phi\left(t_{i}^{-1}\right) & =\left(x_{0}, t_{i_{1}}^{\epsilon_{1}}, \ldots, t_{i_{n-1}}^{\epsilon_{n-1}}, x_{n-1} \phi_{i}\left(x_{n}\right)\right) \Phi\left(t_{i}^{-1}\right) \\
& =\left(x_{0}, t_{i_{1}}^{\epsilon_{1}}, \ldots, t_{i_{n-1}}^{\epsilon_{n-1}}, y_{n-1}, t_{i}^{-1}, \phi_{i}^{-1}(b)\right),
\end{aligned}
$$

where $x_{n-1} \phi_{i}\left(x_{n}\right)=y_{n-1} b, y_{n-1}$ is a $\sim_{B_{i}}$ representative and $b \in B_{i}$ with $y_{n-1}^{-1} y_{n-1}=b b^{-1}$. Then $y_{n-1}=x_{n-1} \phi_{i}\left(x_{n}\right) b^{-1}$. Note that

$$
\begin{aligned}
\left(x_{n-1} \phi_{i}\left(x_{n}\right)\right)^{-1}\left(x_{n-1} \phi_{i}\left(x_{n}\right)\right) & =\phi_{i}\left(x_{n}^{-1}\right) x_{n-1}^{-1} x_{n-1} \phi_{i}\left(x_{n}\right) \\
& =\phi_{i}\left(x_{n}^{-1}\right) \phi_{i}\left(x_{n} x_{n}^{-1}\right) \phi_{i}\left(x_{n}\right)=\phi_{i}\left(x_{n}^{-1} x_{n}\right) .
\end{aligned}
$$


Since $x_{n-1} \phi_{i}\left(x_{n}\right)=y_{n-1} b$, we have

$$
\left(x_{n-1} \phi_{i}\left(x_{n}\right)\right)^{-1}\left(x_{n-1} \phi_{i}\left(x_{n}\right)\right)=\left(y_{n-1} b\right)^{-1}\left(y_{n-1} b\right)=b^{-1} y_{n-1}^{-1} y_{n-1} b=b^{-1} b .
$$

It follows that $b^{-1} b=\phi_{i}\left(x_{n}^{-1} x_{n}\right)$. Then we have

$$
\begin{aligned}
\left(\phi_{i}\left(x_{n}\right) b^{-1}\right)\left(\phi_{i}\left(x_{n}\right) b^{-1}\right)^{-1} & =\phi_{i}\left(x_{n}\right) b^{-1} b \phi_{i}\left(x_{n}^{-1}\right) \\
& =\phi_{i}\left(x_{n}\right) \phi_{i}\left(x_{n}^{-1} x_{n}\right) \phi_{i}\left(x_{n}^{-1}\right)=\phi_{i}\left(x_{n} x_{n}^{-1}\right)=x_{n-1}^{-1} x_{n-1} .
\end{aligned}
$$

Note that $x_{n-1}^{-1} x_{n-1} \in E\left(B_{i}\right), y_{n-1}^{-1} y_{n-1}=b b^{-1} \in E\left(B_{i}\right)$ and $\phi_{i}\left(x_{n}\right) b^{-1} \in B_{i}$. Hence, (E2) holds for $x_{n-1}$ and $y_{n-1}$, and so, $x_{n-1} \sim_{B_{i}} y_{n-1}$. Since both $x_{n-1}$ and $y_{n-1}$ are $\sim_{B_{i}}$ representative, we have $x_{n-1}=y_{n-1}$. By $(\mathrm{Cl})$, we have $\phi_{i}^{-1}\left(x_{n-1}^{-1} x_{n-1}\right)=x_{n} x_{n}^{-1}$. Then $\phi_{i}\left(x_{n}\right)=x_{n-1}^{-1} x_{n-1} \phi_{i}\left(x_{n}\right)=y_{n-1}^{-1} y_{n-1} b=b$, and so, $\phi_{i}^{-1}(b)=x_{n}$. Consequently, we have $X \Phi\left(t_{i}\right) \Phi\left(t_{i}^{-1}\right)=X$.

Next suppose that (2.10) holds. Then we have

$$
X \Phi\left(t_{i}\right)=\left(x_{0}, t_{i_{1}}^{\epsilon_{1}}, \ldots, t_{i_{n-1}}^{\epsilon_{n-1}}, x_{n-1}, t_{i_{n}}^{\epsilon_{n}}, y_{n}, t_{i}, \phi_{i}(a)\right),
$$

where $x_{n}=y_{n} a$ and $y_{n}$ is a $\sim_{A_{i}}$ representative with $y_{n}^{-1} y_{n}=a a^{-1}$ and $a \in A_{i}$. Remark that $t_{i} \phi_{i}(a) t_{i}^{-1}$ is a pinch. Hence, we have

$$
\begin{aligned}
X \Phi\left(t_{i}\right) \Phi\left(t_{i}^{-1}\right) & =\left(x_{0}, t_{i_{1}}^{\epsilon_{1}}, \ldots, t_{i_{n-1}}^{\epsilon_{n-1}}, x_{n-1}, t_{i_{n}}^{\epsilon_{n}}, y_{n}, t_{i}, \phi_{i}(a)\right) \Phi\left(t_{i}^{-1}\right) \\
& =\left(x_{0}, t_{i_{1}}^{\epsilon_{1}}, \ldots, t_{i_{n-1}}^{\epsilon_{n-1}}, x_{n-1}, t_{i_{n}}^{\epsilon_{n}}, y_{n} \phi_{i}^{-1}\left(\phi_{i}(a)\right)\right) \\
& =\left(x_{0}, t_{i_{1}}^{\epsilon_{1}}, \ldots, t_{i_{n-1}}^{\epsilon_{n-1}}, x_{n-1}, t_{i_{n}}^{\epsilon_{n}}, y_{n} a\right) \\
& =\left(x_{0}, t_{i_{1}}^{\epsilon_{1}}, \ldots, t_{i_{n-1}}^{\epsilon_{n-1}}, x_{n-1}, t_{i_{n}}^{\epsilon_{n}}, x_{n}\right)=X .
\end{aligned}
$$

Consequently, we have $X \Phi\left(t_{i}\right) \Phi\left(t_{i}^{-1}\right)=X$ in both (2.9) and (2.10). It follows that

$$
\Phi\left(t_{i}\right) \Phi\left(t_{i}^{-1}\right)=i d_{\operatorname{Dom}\left(\Phi\left(t_{i}\right)\right)}=i d_{\operatorname{Dom}\left(\Phi\left(e_{i}\right)\right)}=\Phi\left(e_{i}\right) .
$$

We have proved (2.13). Similarly, we can prove (2.14). This implies that $\Phi\left(t_{i}^{-1}\right)=$ $\Phi\left(t_{i}\right)^{-1}$ in $\operatorname{Sym}(\mathrm{N})$ for every $i \in I$.

Next we prove the relation (2.15). Take $a$ from $A_{i}$, where $i \in I$. Note that

$$
\operatorname{Ran}(\Phi(a))=\left\{X \in N \mid \mathbf{z}(X) \in S a^{-1} a\right\}
$$

and

$$
\operatorname{Dom}\left(\Phi\left(t_{i}\right)\right)=\left\{X \in N \mid \mathbf{z}(X) \in S e_{i}\right\} .
$$

Since $a \in A_{i} \subset e_{i} S e_{i}$, we have $a^{-1} a \leq e_{i}$ and $a a^{-1} \leq e_{i}$. Then we have

$$
\begin{aligned}
\operatorname{Ran}(\Phi(a)) \cap \operatorname{Dom}\left(\Phi\left(t_{i}\right)\right) & =\left\{X \in N \mid z(X) \in S a^{-1} a \cap S e_{i}\right\} \\
& =\left\{X \in N \mid z(X) \in S a^{-1} a\right\}=\operatorname{Ran}(\Phi(a)) .
\end{aligned}
$$


Hence

$$
\begin{aligned}
\operatorname{Dom}\left(\Phi(a) \Phi\left(t_{i}\right)\right) & =\left(\operatorname{Ran}(\Phi(a)) \cap \operatorname{Dom}\left(\Phi\left(t_{i}\right)\right)\right) \Phi(a)^{-1}=(\operatorname{Ran}(\Phi(a))) \Phi(a)^{-1} \\
& =\operatorname{Dom}(\Phi(a))=\left\{X \in N \mid \mathbf{z}(X) \in S a a^{-1}\right\}
\end{aligned}
$$

Since

$$
\operatorname{Ran}\left(\Phi\left(t_{i}^{-1}\right)\right)=\left\{X \in \mathbf{N} \mid \mathbf{z}(X) \in S e_{i}\right\}
$$

then

$$
\begin{aligned}
\operatorname{Ran}\left(\Phi\left(t_{i}^{-1}\right)\right) \cap \operatorname{Dom}\left(\Phi(a) \Phi\left(t_{i}\right)\right) & =\left\{X \in \mathbf{N} \mid \mathbf{z}(X) \in S a a^{-1} \cap S e_{i}\right\} \\
& =\left\{X \in N \mid z(X) \in S a a^{-1}\right\}
\end{aligned}
$$

since $a a^{-1} \leq e_{i}$. Recall that $\Phi\left(t_{i}^{-1}\right)=\Phi\left(t_{i}\right)^{-1}$ in $\operatorname{Sym}(\mathrm{N})$. Then it is easy to see that

$$
\begin{aligned}
\operatorname{Dom}\left(\Phi\left(t_{i}^{-1}\right) \Phi(a) \Phi\left(t_{i}\right)\right) & =\left\{X \in \mathbf{N} \mid \mathbf{z}(X) \in S a a^{-1}\right\} \Phi\left(t_{i}^{-1}\right)^{-1} \\
& =\left\{X \in \mathbf{N} \mid \mathbf{z}(X) \in S a a^{-1}\right\} \Phi\left(t_{i}\right) \\
& =\left\{X \in \mathbf{N} \mid \mathbf{z}(X) \in S \phi_{i}\left(a a^{-1}\right)\right\}
\end{aligned}
$$

On the other hand, we have

$$
\begin{aligned}
\operatorname{Dom}\left(\Phi\left(\phi_{i}(a)\right)\right) & =\left\{X \in \mathbf{N} \mid \mathbf{z}(X) \in S \phi_{i}(a)\left(\phi_{i}(a)\right)^{-1}\right\} \\
& =\left\{X \in \mathbf{N} \mid \mathbf{z}(X) \in S \phi_{i}\left(a a^{-1}\right)\right\} .
\end{aligned}
$$

Hence, $\operatorname{Dom}\left(\Phi\left(t_{i}^{-1}\right) \Phi(a) \Phi\left(t_{i}\right)\right)=\operatorname{Dom}\left(\Phi\left(\phi_{i}(a)\right)\right)$.

Take a normal sequence $X$ from $\operatorname{Dom}\left(\Phi\left(t_{i}^{-1}\right) \Phi(a) \Phi\left(t_{i}\right)\right)$. Suppose that $X=$ $\left(x_{0}, t_{i_{1}}^{\epsilon_{1}}, x_{1}, \ldots, x_{n-2}, t_{i_{n-1}}^{\epsilon_{n-1}}, x_{n-1}, t_{i_{n}}^{\epsilon_{n}}, x_{n}\right)$. In computing $X \Phi\left(t_{i}^{-1}\right)$ for $X$ and $t_{i}^{-1}$, there are two possible cases; (2.11) and (2.12).

Suppose that (2.11) holds. Then we have $i=i_{n}, \epsilon_{n}=1$ and $x_{n} \in B_{i_{n}}=B_{i}$. Remark that it cannot happen that $x_{n-1} \in A_{i}, i=i_{n}$ and $\epsilon_{n-1}=-1$ since $X \in \mathrm{N}$. Then $\phi_{i}\left(x_{n-1}^{-1} x_{n-1}\right)=\phi_{i_{n}}\left(x_{n-1}^{-1} x_{n-1}\right)=x_{n} x_{x}^{-1}$ by $(\mathrm{C} 1)$. We have

$$
X \Phi\left(t_{i}^{-1}\right)=\left(x_{0}, t_{i_{1}}^{\epsilon_{1}}, x_{1}, \ldots, x_{n-2}, t_{i_{n-1}}^{\epsilon_{n-1}}, x_{n-1} \phi_{i}^{-1}\left(x_{n}\right)\right) .
$$

Then we have

$$
X \Phi\left(t_{i}^{-1}\right) \Phi(a)=\left(x_{0}, t_{i_{i}}^{\epsilon_{1}}, x_{1}, \ldots, x_{n-2}, t_{i_{n-1}}^{\epsilon_{n-1}}, x_{n-1} \phi_{i}^{-1}\left(x_{n}\right) a\right) .
$$

By the remark above $t_{i_{n-1}}^{\epsilon_{n-1}}, x_{n-1} \phi_{i}^{-1}\left(x_{n}\right) a t_{i}$ is not a pinch, and so, we have

$$
X \Phi\left(t_{i}^{-1}\right) \Phi(a) \Phi\left(t_{i}\right)=\left(x_{0}, t_{i_{1}}^{\epsilon_{1}}, x_{1}, \ldots, x_{n-2}, t_{i_{n-1}}^{\epsilon_{n-1}}, y_{n-1}, t_{i}, \phi_{i}(c)\right),
$$

where $y_{n-1}$ is a $\sim_{A_{i}}$ representative and $y_{n-1}^{-1} y_{n-1}=c c^{-1}, c \in A_{i}$ and $x_{n-1} \phi_{i}^{-1}\left(x_{n}\right) a=$ $y_{n-1} c$. Then both $x_{n-1}$ and $y_{n-1}$ are $\sim_{A_{i}}$ representative. Note that $x_{n-1}^{-1} x_{n-1}$ belongs to $E\left(A_{i}\right)$ by the condition $(\mathrm{Cl})$. Also note that $y_{n-1}^{-1} y_{n-1}$ belongs to $E\left(A_{i}\right)$ since $c \in A_{i}$ and $y_{n-1}^{-1} y_{n-1}=c c^{-1}$. Set $h=\phi_{i}^{-1}\left(x_{n}\right) a c^{-1}$. Then $h$ belongs to $A_{i}$ (as $x_{n} \in B_{i}, a \in A_{i}$ 
and $\left.c \in A_{i}\right)$. Since $x_{n}=\mathbf{z}(X) \in S \phi_{i}\left(a a^{-1}\right)$, we have $x_{n}=s \phi_{i}\left(a a^{-1}\right)$ for some $s \in S$. Then $x_{n} \phi_{i}\left(a a^{-1}\right)=x_{n}$, and so, $\phi_{i}^{-1}\left(x_{n}\right) a a^{-1}=\phi_{i}^{-1}\left(x_{n}\right)$. We have

$$
x_{n-1} h=x_{n-1} \phi_{i}^{-1}\left(x_{n}\right) a c^{-1}=y_{n-1} c c^{-1}=y_{n-1} y_{n-1}^{-1} y_{n-1}=y_{n-1}
$$

and

$$
\begin{aligned}
y_{n-1} h^{-1} & =y_{n-1} c a^{-1} \phi_{i}^{-1}\left(x_{n}^{-1}\right)=x_{n-1} \phi_{i}^{-1}\left(x_{n}\right) a a^{-1} \phi_{i}^{-1}\left(x_{n}^{-1}\right) \\
& =x_{n-1} \phi_{i}^{-1}\left(x_{n}\right) \phi_{i}^{-1}\left(x_{n}^{-1}\right)=x_{n-1} \phi_{i}^{-1}\left(x_{n} x_{n}^{-1}\right)=x_{n-1} x_{n-1}^{-1} x_{n-1}=x_{n-1} .
\end{aligned}
$$

Then $x_{n-1} x_{n-1}^{-1}=y_{n-1} y_{n-1}^{-1}$. Furthermore, we have

$$
\begin{aligned}
x_{n-1}^{-1} y_{n-1} & =x_{n-1}^{-1} x_{n-1} h=x_{n-1}^{-1} x_{n-1} \phi_{i}^{-1}\left(x_{n}\right) a c^{-1} \\
& =\phi_{i}^{-1}\left(x_{n} x_{n}^{-1}\right) \phi_{i}^{-1}\left(x_{n}\right) a c^{-1}=\phi_{i}^{-1}\left(x_{n}\right) a c^{-1}=h \in A_{i} .
\end{aligned}
$$

It follows that $x_{n-1} \sim_{A_{i}} y_{n-1}$ by Lemma 2.3. Since both $x_{n-1}$ and $y_{n-1}$ are $\sim_{A_{i}}$ representative, we have $x_{n-1}=y_{n-1}$. Since $x_{n-1} \phi_{i}^{-1}\left(x_{n}\right) a=y_{n-1} c$ and $y_{n-1}^{-1} y_{n-1}=$ $c c^{-1}$, we have $c=y_{n-1}^{-1} y_{n-1} c=x_{n-1}^{-1} x_{n-1} \phi_{i}^{-1}\left(x_{n}\right) a=\phi_{i}^{-1}\left(x_{n}\right) a$. Hence, $\phi_{i}(c)=$ $\phi_{i}\left(\phi_{i}^{-1}\left(x_{n}\right) a\right)=x_{n} \phi_{i}(a)$. Consequently, we have

$$
\begin{aligned}
X \Phi\left(t_{i}^{-1}\right) \Phi(a) \Phi\left(t_{i}\right) & =\left(x_{0}, t_{i_{1}}^{\epsilon_{1}}, x_{1}, \ldots, x_{n-2}, t_{i_{n-1}}^{\epsilon_{n-1}}, y_{n-1}, t_{i}, \phi_{i}(c)\right) \\
& =\left(x_{0}, t_{i_{1}}^{\epsilon_{1}}, x_{1}, \ldots, x_{n-2}, t_{i_{n-1}}^{n_{n-1}}, x_{n-1}, t_{i}, x_{n} \phi_{i}(a)\right) \\
& =X \Phi\left(\phi_{i}(a)\right) .
\end{aligned}
$$

Next suppose that (2.12) holds. Then

$$
X \Phi\left(t_{i}^{-1}\right)=\left(x_{0}, t_{i_{1}}^{\epsilon_{1}}, x_{1}, \ldots, x_{n-2}, t_{i_{n-1}}^{\epsilon_{n-1}}, x_{n-1}, t_{i_{n}}^{\epsilon_{n}}, y_{n}, t_{i}^{-1}, \phi_{i}^{-1}(b)\right),
$$

where $x_{n}=y_{n} b$ and $y_{n}$ is a $\sim_{B_{i}}$ representative such that $y_{n}^{-1} y_{n}=b b^{-1}$ and $b \in B_{i}$. Then

$$
X \Phi\left(t_{i}^{-1}\right) \Phi(a)=\left(x_{0}, t_{i_{1}}^{\epsilon_{1}}, x_{1}, \ldots, x_{n-2}, t_{i_{n-1}}^{\epsilon_{n-1}}, x_{n-1}, t_{i_{n}}^{\epsilon_{n}}, y_{n}, t_{i}^{-1}, \phi_{i}^{-1}(b) a\right) .
$$

Since $t_{i}^{-1} \phi_{i}^{-1}(b) a t_{i}$ is a pinch,

$$
\begin{aligned}
X \Phi\left(t_{i}^{-1}\right) \Phi(a) \Phi\left(t_{i}\right) & =\left(x_{0}, t_{i_{1}}^{\epsilon_{1}}, x_{1}, \ldots, x_{n-2}, t_{i_{n-1}}^{\epsilon_{n-1}}, x_{n-1}, t_{i_{n}}^{\epsilon_{n}}, y_{n} \phi_{i}\left(\phi_{i}^{-1}(b) a\right)\right) \\
& =\left(x_{0}, t_{i_{1}}^{\epsilon_{1}}, x_{1}, \ldots, x_{n-2}, t_{i_{n-1}}^{\epsilon_{n}}, x_{n-1}, t_{i_{n}}^{\epsilon_{n}}, y_{n} b \phi_{i}(a)\right) \\
& =\left(x_{0}, t_{i_{1}}^{\epsilon_{1}}, x_{1}, \ldots, x_{n-2}, t_{i_{n-1}}^{\epsilon_{n-1}}, x_{n-1}, t_{i_{n}}^{\epsilon_{n}}, x_{n} \phi_{i}(a)\right) \\
& =X \Phi\left(\phi_{i}(a)\right) .
\end{aligned}
$$

We have proved (2.15).

Consequently, we can extend the mapping $\Phi$ to a homomorphism of $S^{*}$ into $\operatorname{Sym}(\mathrm{N})$ by Proposition 1.1. Now suppose that $x\left(\in S^{*}\right)$ has a normal form $x_{0} t_{i_{1}}^{\epsilon_{1}} x_{1} t_{i_{2}}^{\epsilon_{2}} \cdots t_{i_{n}}^{\epsilon_{n}} x_{n}$. Then the sequence $\left(x_{0}, t_{i_{1}}^{\epsilon_{1}}, x_{1}, t_{i_{2}}^{\epsilon_{2}}, \ldots, t_{i_{n}}^{\epsilon_{n}}, x_{n}\right)$ is normal, and so, it belongs to N. Set 
$e=x x^{-1}$. By Lemma 2.6, we have $e=x_{0} x_{0}^{-1}$. Then it is easy to see that the normal sequence $(e)$ is in $\operatorname{Dom}(\Phi(x))$ and

$$
\begin{aligned}
(e) \Phi(x) & =(e) \Phi\left(x_{0} t_{i_{1}}^{\epsilon_{1}} x_{1} t_{i_{2}}^{\epsilon_{2}} \cdots t_{i_{n}}^{\epsilon_{n}} x_{n}\right) \\
& =\left(x_{0}, t_{i_{1}}^{\epsilon_{1}}, x_{1}, t_{i_{2}}^{\epsilon_{2}}, \ldots, t_{i_{n}}^{\epsilon_{n}}, x_{n}\right) .
\end{aligned}
$$

This implies the uniqueness of a normal form of $x$.

We have completed the proof of Theorem 2.7. A similar normal form is obtained for a full HNN extension of an inverse monoid with one stable letter and a locally full HNN extension of a semilattice in [15] and [17], respectively.

\section{Applications of the normal form theorem}

An immediate consequence of Theorem 2.7 is that an inverse semigroup $S$ is strongly embedded into any locally full HNN extension $S^{*}$. Note that this follows from Proposition 1.2. Another easy consequence is that a formation of a locally full HNN extension yields no new idempotent. Conversely, if an HNN extension of an inverse semigroup has the same semilattice of idempotents as the original semigroup, then it must be locally full as we will see next.

COROLlaRY 3.1. An inverse semigroup $S$ is strongly embedded into a locally full HNN extension $S^{*}$ and $E\left(S^{*}\right)=E(S)$. Conversely, if $S^{*}$ is an HNN extension of $S$ with the property that $E\left(S^{*}\right)=E(S)$, then $S^{*}$ is a locally full $\mathrm{HNN}$ extension of $S$.

PROOF. Every element $x$ of $S$ has the normal form $x$ since the sequence $(x)$ satisfies the conditions (C1), (C2) and (C3). It follows that $S$ can be naturally embedded into $S^{*}$.

Take $x \in t_{i}^{-1} S t_{i} \cap S$. Then $x=t_{i}^{-1} s t_{i}$, where $s \in S$. Using Lemma 2.8 we may assume that $t_{i}^{-1} s t_{i}$ is a form. If $t_{i}^{-1} s t_{i}$ is not a pinch, then $x$ has the normal form $s_{0} t_{i}^{-1} s_{1} t_{i}$, where $s_{0}, s_{1} \in S$. On the other hand, since $x \in S, x$ is in the normal form. This contradicts the uniqueness of the normal form. Thus, $t_{i}^{-1} s t_{i}$ must be a pinch, and so, $s \in A_{i}$ and $t_{i}^{-1} s t_{i}=\phi_{i}(a) \in B_{i}$. It follows that $t_{i}^{-1} S t_{i} \cap S=B_{i}$ and that $S$ is strongly embedded in $S^{*}$.

Take any idempotent $e$ from $E\left(S^{*}\right)$. Suppose that $e$ has the normal form $x_{0} t_{i_{1}}^{\epsilon_{1}} x_{1} t_{i_{2}}^{\epsilon_{2}} \ldots$ $t_{i_{n}}^{\epsilon_{n}} x_{n}$. By Lemma 2.6, we have

$$
e=e e^{-1}=\left(x_{0} t_{i_{1}}^{\epsilon_{1}} x_{1} t_{i_{2}}^{\epsilon_{2}} \cdots t_{i_{n}}^{\epsilon_{n}} x_{n}\right)\left(x_{0} t_{i_{1}}^{\epsilon_{1}} x_{1} t_{i_{2}}^{\epsilon_{2}} \cdots t_{i_{n}}^{\epsilon_{n}} x_{n}\right)^{-1}=x_{0} x_{0}^{-1} \in E(S)
$$

since $x_{0} \in S$. This shows that $E\left(S^{*}\right) \subset E(S)$. Clearly $E(S) \subset E\left(S^{*}\right)$ since $S$ is embedded into $S^{*}$. 
Conversely, we assume that the semilattice $E\left(S^{*}\right)$ is equal to $E(S)$. We show that each $E\left(A_{i}\right)$ coincides with $E\left(e_{i} S e_{i}\right)=E(S) e_{i}$. Take any $e \in E(S) e_{i}$. The element $t_{i}^{-1} e t_{i}$ is an idempotent of $S^{*}$. Since $E\left(S^{*}\right)=E(S), t_{i}^{-1} e t_{i}$ is in $E(S)$. Then $t_{i}^{-1} e t_{i} \in E(S) \cap t_{i}^{-1}\left(E(S) e_{i}\right) t_{i} \subset S \cap t_{i}^{-1} S t_{i}$. On the other hand, we have $S \cap t_{i}^{-1} S t_{i}=$ $t_{i}^{-1} A_{i} t_{i}=B_{i}$ by (1.6). It follows that $t_{i}^{-1} e t_{i} \in t_{i}^{-1} A_{i} t_{i}$ and that $e=t_{i} t_{i}^{-1} e t_{i} t_{i}^{-1} \subset$ $t_{i} t_{i}^{-1} A_{i} t_{i} t_{i}^{-1} \subset A_{i}$ (as $t_{i} t_{i}^{-1}=e_{i} \in A_{i}$ and $\left.e \leq e_{i}\right)$. Therefore, $E\left(A_{i}\right)=E(S) e_{i}$, and hence, $S^{*}$ is a locally full HNN extension of $S$.

COROLLARY 3.2. If an element $x$ of $S^{*}$ has a reduced form

$$
x_{0} t_{i_{1}}^{\epsilon_{1}} x_{1} t_{i_{2}}^{\epsilon_{2}} \cdots t_{i_{n}}^{\epsilon_{n}} x_{n}
$$

where $n \geq 1$, then $x \notin E\left(S^{*}\right)=E(S)$.

ProOF. By Lemma 2.10, if the element $x$ has a reduced form (3.1), then $x$ has the normal form $u_{0} t_{i_{1}}^{\epsilon_{1}} u_{1} t_{i_{2}}^{\epsilon_{2}} \cdots t_{i_{n}}^{\epsilon_{n}} u_{n}$ for some $u_{0}, u_{1}, \ldots, u_{n}$ in $S$. Since $E\left(S^{*}\right)=E(S)$ by Corollary 3.1, every idempotent $e$ has the normal form $e$. Since the expression is unique, we have $x \notin E(S)$.

Note that Corollary 3.2 is considered as a generalization of Britton's lemma in group theory $([3,10])$.

Some algebraic properties are not preserved under a formation of a locally full HNN extension. For examples, the property of being a finite semigroup, being commutative, being free, being E-unitary, being factorizable, being combinatorial, being fundamental, being congruence-free, being completely semisimple or having a zero element is not necessarily preserved under a formation of a locally full HNN extension.

On the other hand, we show that several algebraic properties are preserved under a formation of a locally full HNN extension. It is easy to see that an HNN extension of a monoid (group) is always a monoid (group).

COROLLARY 3.3. Let $S$ be listed below. Then a locally full $\mathrm{HNN}$ extension $S^{*}$ satisfies $\mathscr{P}$ if $S$ does.

(1) Having finitely many idempotents.

(2) Being torsion-free.

(3) Being bisimple.

(4) Being simple.

Proof. (1) By Corollary 3.1, $E(S)=E\left(S^{*}\right)$ and so the HNN extension $S^{*}$ has finitely many idempotents if $S$ does so. (2) Using Theorem 2.7 , we can easily show that $S^{*}$ is torsion-free if $S$ is. (3) We note that an inverse semigroup is bisimple if 
and only if all idempotents are $\mathscr{D}$-related. By Corollary 3.1 , all idempotents of $S^{*}$ are $\mathscr{D}$-related in $S^{*}$ if all idempotents of $S$ are $\mathscr{D}$-related in $S$. (4) We note that an inverse semigroup is simple if and only if all idempotents are $\mathscr{J}$-related. By Corollary 3.1 , all idempotents of $S^{*}$ are $\mathscr{J}$-related in $S^{*}$ if all idempotents of $S$ are $\mathscr{J}$-related in $S$.

Under a certain condition on associated inverse submonoids $A_{i}$ and $B_{i}(i \in I)$, algebraic properties are preserved.

COROLLARY 3.4. Let $S$ be an E-unitary inverse semigroup. Suppose that $S^{*}$ is a locally full $\mathrm{HNN}$ extension of $S$ associated with $\phi_{i}: A_{i} \rightarrow B_{i}(i \in I)$. If $A_{i}$ and $B_{i}$ are closed in $S$ for every $i \in I$, then $S^{*}$ is $E$-unitary. '

Proof. Suppose that $S^{*}$ is not E-unitary. There exist $e \in E\left(S^{*}\right)=E(S)$ and $x \in S^{*} \backslash E(S)$ such that $e \leq x$. By Theorem 2.7, $x$ has the normal form $x_{0} t_{i_{1}}^{\epsilon_{1}} x_{1} t_{i_{2}}^{\epsilon_{2}} \cdots x_{n-1} t_{i_{n}}^{\epsilon_{n}} x_{n}$, where either $n \geq 1$ or $x_{0} \notin E(S)$. Since $e \leq x$, there exists $f \in E(S)$ such that $f x=e$. Then it is easy to see that

$$
f x=f x_{0} t_{i_{1}}^{\epsilon_{1}} x_{1} t_{i_{2}}^{\epsilon_{2}} \cdots x_{n-1} t_{i_{n}}^{\epsilon_{n}} x_{n}=x_{0}^{\prime} t_{i_{1}}^{\epsilon_{1}} x_{1}^{\prime} t_{i_{2}}^{\epsilon_{2}} \cdots x_{n-1}^{\prime} t_{i_{n}}^{\epsilon_{n}} x_{n}^{\prime},
$$

for some $x_{0}^{\prime}, x_{1}^{\prime}, \ldots, x_{n}^{\prime}$ in $S$ with $x_{i}^{\prime} \leq x_{i}$ for every $i$. By Lemma 2.8, we may assume that $e=f x$ has the form $x_{0}^{\prime} t_{i_{1}}^{\epsilon_{1}} x_{1}^{\prime} t_{i_{2}}^{\epsilon_{2}} \cdots x_{n-1}^{\prime} t_{i_{n}}^{\epsilon_{n}} x_{n}^{\prime}$. Then either $x_{i}^{\prime} \in A_{i}$ or $x_{i}^{\prime} \in B_{i}$ holds for some $i$ by Corollary 3.2. On the other hand, we have $x_{i} \notin A_{i}$ and $x_{i} \notin B_{i}$. This contradicts the fact that $A_{i}$ (or $B_{i}$ ) is closed in $S$. Consequently $S^{*}$ is E-unitary.

Similarly any locally full HNN extension of an F-inverse monoid associated with closed inverse submonoids is F-inverse. We next discuss the word problem for a locally full HNN extension.

LEMMA 3.5. Let $S$ be an inverse semigroup, and let $S^{*}$ be a locally full $\mathrm{HNN}$ extension of $S$ associated with the isomorphisms $\phi_{i}: A_{i} \rightarrow B_{i}(i \in I)$. If the membership problems for the semilattice $E(S), A_{i}$ and $B_{i}(i \in I)$ in $S$ are solvable and the isomorphisms $\phi_{i}$ and $\phi_{i}^{-1}$ are effectively computable $(i \in I)$, then the membership problem for $E(S)$ in $S^{*}$ is solvable.

PROOF. For a given word $w$ on generators of $S^{*}$, we can effectively obtain a reduced form for $w$ by Lemma 2.8 and Lemma 2.9. Suppose that the reduced form for $w$ is $x_{0} t_{i_{1}}^{\epsilon_{1}} x_{1} t_{i_{2}}^{\epsilon_{2}} \cdots t_{i_{n}}^{\epsilon_{n}} x_{n}$. If $n>0$, then we can conclude that $w \notin E\left(S^{*}\right)$. If $n=0, w$ is written as a word on generators of $S$. We use the algorithm for the membership problem for $E(S)$ in $S$ to determine whether or not $w$ is in $E(S)$. If so, we can conclude that $w \in E\left(S^{*}\right)$, otherwise $w \notin E\left(S^{*}\right)$. 
THEOREM 3.6. If the word problem for $S$ is solvable and $\phi_{i}$ and $\phi_{i}^{-1}$ are effectively computable for every $i \in I$ and the membership problems for $A_{i}$ and $B_{i}$ in $S$ are solvable for every $i \in I$, then the word problem for $S^{*}$ is solvable.

PROOF. Let $w_{1}$ and $w_{2}$ be given words on generators of $S^{*}$. We note that $w_{1}=w_{2}$ if and only if $w_{1} w_{1}^{-1}=w_{2} w_{2}^{-1}$ and $w_{2}^{-1} w_{1} \in E\left(S^{*}\right)=E(S)$. For, if $w_{1} w_{1}^{-1}=w_{2} w_{2}^{-1}$ and $w_{2}^{-1} w_{1} \in E(S)$, then we have

$$
\begin{aligned}
w_{1} & =w_{1} w_{1}^{-1} w_{1}=w_{2} w_{2}^{-1} w_{1}=w_{2} w_{2}^{-1} w_{1} w_{2}^{-1} w_{1} \\
& =w_{2} w_{2}^{-1} w_{1} w_{1}^{-1} w_{2} \quad\left(\text { as } w_{2}^{-1} w_{1}=\left(w_{2}^{-1} w_{1}\right)^{-1}=w_{1}^{-1} w_{2}\right) \\
& =w_{2} w_{2}^{-1} w_{2} w_{2}^{-1} w_{2}=w_{2} .
\end{aligned}
$$

Hence, to solve the word problem for $S^{*}$ it is enough to decide for given words $w_{1}$ and $w_{2}$ whether or not $w_{1} w_{1}^{-1}=w_{2} w_{2}^{-1}$ and $w_{2}^{-1} w_{1} \in E(S)$.

Since the word problem for $S$ is solvable, we can effectively determine whether or not $w=w^{2}$ for a given word $w$ on the generators of $S$. Therefore, the membership problem for $E(S)$ in $S$ is solvable. By Lemma 3.5 , we can effectively determine whether or not $w_{2}^{-1} w_{1} \in E(S)$. By Lemma 2.8 and Lemma 2.9, we can effectively find reduced form $e_{1}$ and $e_{2}$ for $w_{1} w_{1}^{-1}$ and $w_{2} w_{2}^{-1}$, respectively. Then $w_{1} w_{1}^{-1}$ and $w_{2} w_{2}^{-1}$ are written as words on the generators of $S$. By Corollary 3.1, $e_{1}$ and $e_{2}$ are in $E(S)$. Since the word problem for $S$ is solvable, we can effectively decide whether or not $e_{1}=e_{2}$ in $S$. Consequently, the word problem for $S^{*}$ is solvable.

COROLLARY 3.7. A locally full HNN extension of a finite inverse semigroup has solvable word problem.

PROOF. Obviously the word problem for a finite inverse semigroup is solvable, the membership problems for inverse subsemigroups are solvable and the partial isomorphisms are effectively computable.

Margolis and Meakin [11] solved the word problem for an idempotent pure image of a free inverse monoid using Rabin's tree theorem. Using their result, the author showed that any HNN extension of a finite semilattice has solvable word problem ([17]). Note that any idempotent pure image of a free inverse semigroup is an HNN extension of a semilattice. However, a finitely generated idempotent pure image of a free inverse semigroup does not necessarily have solvable word problem. Jajcayova [8] solved the word problem for an HNN extension of a free inverse semigroup associated with finitely generated inverse subsemigroups using graphical methods. It is not known whether or not any HNN extension of a finite inverse semigroup has solvable word problem. We remark that Cherubini, Meakin and Piochi [2] proved the solvability of the word problem for an amalgamated free product of free inverse semigroups associated with finitely generated inverse subsemigroups. 


\section{Action of a maximal subgroup on a tree}

Haataja, Margolis and Meakin [4] employed the Bass-Serre theory to obtain a group presentation of a maximal subgroup of a full amalgamated free product of regular semigroups. See $[1,8,12,15]$ for similar applications of the Bass-Serre theory to find presentations of maximal subgroups of free constructions of inverse semigroups. We obtain a group presentation of a maximal subgroup of a locally full HNN extension using the Bass-Serre theory. The reader is referred to $[3,14]$ for the detail of the Bass-Serre theory.

Let $S^{*}$ be the locally full HNN extension given by (2.2). Let $e$ be an idempotent in $E\left(S^{*}\right)=E(S)$. We denote the maximal subgroup (grdup $\mathscr{H}$-class) of $S^{*}$ containing $e$ by $S_{e}^{*}$. We construct a graph $X_{e}$ on which $S_{e}^{*}$ acts without inversion.

4.1. Forest $X \quad$ Let $S$ be an inverse semigroup and $H$ an inverse subsemigroup of $S$. For any $x$ in $S$ with $x^{-1} x \in E(H)$, we define a coset $x H$ to be

$$
x H=\left\{x h \mid x^{-1} x=h h^{-1}(h \in H)\right\} .
$$

LEMMA 4.1. The coset $x H$ is identical to the $\sim_{H}$ class containing $x$ if $x^{-1} x \in E(H)$.

PROOF. Recall that $\sim_{H}$ is defined in (2.1). Suppose that $x^{-1} x \in E(H)$. Then, if $y \in x H$, then $y=x h$ for some $h \in H$ with $x^{-1} x=h h^{-1}$. By the definition of $\sim_{H}$, we have $x \sim_{H} y$ and so $x H \subset \bar{x}$. Conversely, we take $y \in \bar{x}$. Then, $y=x h$ with $h \in H$ and $x^{-1} x=h h^{-1}$. Hence, $y=x h \in x H$. Thus, $\bar{x} \subset x H$.

LEMMA 4.2. For $x, y \in S$ with $x^{-1} x, y^{-1} y \in E(H), x H=y H$ if and only if $x \mathscr{R} y$ in $S$ and $x^{-1} y \in H$.

PROOF. This is an immediate consequence of Lemma 2.3 and Lemma 4.1.

LEMMA 4.3. Let $x, y \in S$ with $x^{-1} x, y^{-1} y \in E(H)$. If $x H=y H$, then $x \mathscr{R} y$ and $x^{-1} x \mathscr{D} y^{-1} y$ in $H$.

PROOF. By Lemma 4.2, we have $x \mathscr{R} y$ in $S$ and $x^{-1} y \in H$. Let $h=x^{-1} y$. Then we have

$$
h h^{-1}=\left(x^{-1} y\right)\left(x^{-1} y\right)^{-1}=x^{-1}\left(y y^{-1}\right) x=x^{-1}\left(x x^{-1}\right) x=x^{-1} x .
$$

Hence, $h \mathscr{R} x^{-1} x$ in $H$. Similarly we can show $h \mathscr{L} y^{-1} y$ in $H$. Consequently, $x^{-1} x \mathscr{D} y^{-1} y$ in $H$. 


$$
\begin{aligned}
& \mathbf{i}\left(x A_{i}\right)=x S \quad \mathbf{t}\left(x A_{i}\right)=x t_{i} S \quad \mathbf{t}\left(x B_{i}\right)=x t_{i}^{-1} S \quad \mathbf{i}\left(x B_{i}\right)=x S \\
& \mathrm{O} \longrightarrow \mathrm{O} \\
& x A_{i} \quad x B_{i}
\end{aligned}
$$

FIGURE 1. Edge and initial and terminal vertex
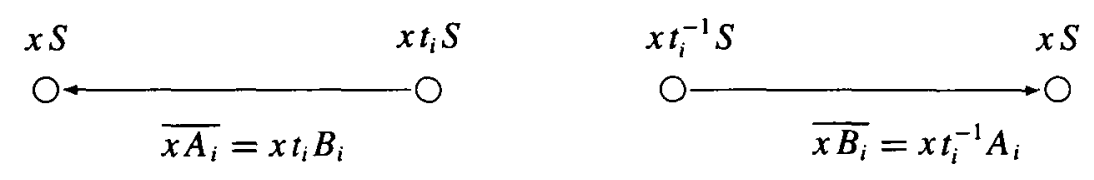

FiguRE 2. Inverse edge

The graph $X$ consists of the set $\operatorname{Vert}(X)$ of vertices and the set $\operatorname{Edge}(X)$ of edges. The set $\operatorname{Vert}(X)$ consists of cosets $x S$ :

$$
\operatorname{Vert}(X)=\left\{x S \mid x \in S^{*}\right\}
$$

Note that $x^{-1} x \in E\left(S^{*}\right)=E(S)$ for every $x \in S^{*}$. The set Edge ${ }_{+}(X)$ of the positively oriented edges of $X$ consists of cosets $x A_{i}(i \in I)$ :

$$
\text { Edge }_{+}(X)=\left\{x A_{i} \mid x \in S^{*}, x^{-1} x \leq e_{i}=t_{i} t_{i}^{-1} \text { for } i \in I\right\} .
$$

The set Edge $(X)$ of the negatively oriented edges of $X$ consists of $\operatorname{cosets} x B_{i}(i \in I)$ :

$$
\text { Edge }_{-}(X)=\left\{x B_{i} \mid x \in S^{*}, x^{-1} x \leq f_{i}=t_{i}^{-1} t_{i} \text { for } i \in I\right\} .
$$

We make a convention that $x A_{i}$ and $x A_{j}$ are distinct for distinct $i$ and $j$ even if $A_{i}$ coincides with $A_{j}$, and similarly, $x B_{i}$ and $x B_{j}$ are distinct for distinct $i$ and $j$ even if $B_{i}$ coincides with $B_{j}$. The setEdge $(X)$ is the union of Edge ${ }_{+}(X)$ and Edge $(X)$ :

$$
\operatorname{Edge}(X)=\operatorname{Edge}_{+}(X) \cup \operatorname{Edge}_{-}(X)
$$

Here we make a convention that $\operatorname{Edge}_{+}(X)$ and Edge $(X)$ are disjoint (even if $A_{i}=B_{j}$ for some $i$ and $j$ ).

We define the initial vertex, the terminal vertex and the inverse edge. The initial vertex of $x A_{i}$ and $x B_{i}$ are defined by

$$
\mathbf{i}\left(x A_{i}\right)=x S, \quad \mathbf{i}\left(x B_{i}\right)=x S .
$$




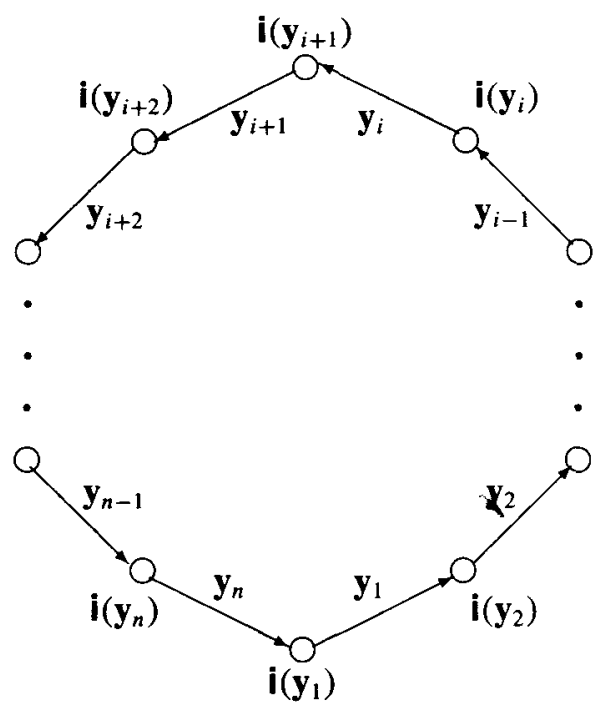

FIgURE 3. Circuit

The terminal vertex of $x A_{i}$ and $x B_{i}$ are defined by

$$
\mathbf{t}\left(x A_{i}\right)=x t_{i} S, \quad \mathbf{t}\left(x B_{i}\right)=x t_{i}^{-1} S .
$$

The inverse edge of $x A_{i}$ and $x B_{i}$ are defined by

$$
\overline{x A_{i}}=x t_{i} B_{i}, \quad \overline{x B_{i}}=x t_{i}^{-1} A_{i}
$$

See Figure 1 and Figure 2. It is routine to verify that $\operatorname{Vert}(X)$ and $\operatorname{Edge}(X)$ forms a graph in the sense of Serre [14].

THEOREM 4.4. The graph $X$ defined by (4.1)-(4.7) is a forest.

PrOOF. Suppose that $X$ is not a forest. Then there exists a circuit without backtracking. Suppose that the edges $\mathbf{y}_{1}, \mathbf{y}_{2}, \ldots, \mathbf{y}_{n}(n \geq 1)$ form a circuit without backtracking in this order. See Figure 3. We have $\mathbf{t}\left(\mathbf{y}_{k}\right)=\mathbf{i}\left(\mathbf{y}_{k+1}\right)$ for each $k=1,2, \ldots, n-1$ and $\mathbf{t}\left(\mathbf{y}_{n}\right)=\mathbf{i}\left(\mathbf{y}_{1}\right)$. We also have $\mathbf{y}_{k} \neq \overline{\mathbf{y}_{k+1}}$ for $k=1,2, \ldots, n-1$ and $\mathbf{y}_{1} \neq \overline{\mathbf{y}_{n}}$.

We assume that $n \geq 2$ and derive a contradiction. In the case of $n=1$, we can similarly derive a contradiction and so we do not discuss the case. If $\mathbf{y}_{k} \in \operatorname{Edge}_{+}(X)$, then we have $\mathbf{y}_{k}=z_{k-1} A_{i_{k}}$ for some $z_{k-1} \in S^{*}$ with $z_{k-1}^{-1} z_{k-1} \in A_{i_{k}}$. Then we define $\epsilon_{k}$ to be 1. We note that $\mathbf{i}\left(\mathbf{y}_{k}\right)=z_{k-1} S$ and $\mathbf{t}\left(\mathbf{y}_{k}\right)=z_{k-1} t_{i_{k}} S=z_{k-1} t_{i_{k}}^{\epsilon_{k}} S$. If $\mathbf{y}_{k} \in$ Edge_$(X)$, then we have $\mathbf{y}_{k}=z_{k-1} B_{i_{k}}$ for some $z_{k-1} \in S^{*}$ with $z_{k-1}^{-1} z_{k-1} \in B_{i_{k}}$. Then we define $\epsilon_{k}$ to be -1 . We note that $\mathbf{i}\left(\mathbf{y}_{k}\right)=z_{k-1} S$ and $\mathbf{t}\left(\mathbf{y}_{k}\right)=z_{k-1} t_{i_{k}}^{-1} S=z_{k-1} t_{i_{k}} S$. In the both cases, we have $\mathbf{i}\left(\mathbf{y}_{k}\right)=z_{k-1} S$ and $\mathbf{t}\left(\mathbf{y}_{k}\right)=z_{k-1} t_{i_{k}}^{\epsilon_{k}} S$ for every $k=1,2, \ldots, n$. 
Since $\mathbf{t}\left(\mathbf{y}_{k}\right)=\mathbf{i}\left(\mathbf{y}_{k+1}\right)$ for every $k=1,2, \ldots, n-1$, we have $z_{k-1} t_{i_{k}}^{\epsilon_{k}} S=z_{k} S$. There exists $x_{k} \in S$ such that $z_{k}=z_{k-1} t_{i_{k}}^{\epsilon_{k}} x_{k}$ with $\left(z_{k-1} t_{i_{k}}^{\epsilon_{k}}\right)^{-1}\left(z_{k-1} t_{i_{k}}^{\epsilon_{k}}\right)=x_{k} x_{k}^{-1}$ for every $k=1,2, \ldots, n-1$. Similarly there exists $x_{n} \in S$ such that $z_{0}=z_{n-1} t_{i_{n}}^{\epsilon_{n}} x_{n}$ with $\left(z_{n-1} t_{i_{n}}^{\epsilon_{n}}\right)^{-1}\left(z_{n-1} t_{i_{n}}^{\epsilon_{n}}\right)=x_{n} x_{n}^{-1}$, since we have $\mathbf{t}\left(\mathbf{y}_{n}\right)=\mathbf{i}\left(\mathbf{y}_{1}\right)$. Then we have

$$
z_{k}^{-1} z_{k}=x_{k}^{-1} t_{i_{k}}^{-\epsilon_{k}} z_{k-1}^{-1} z_{k-1} t_{i_{k}}^{\epsilon_{k}} x_{k}=x_{k}^{-1} x_{k} x_{k}^{-1} x_{k}=x_{k}^{-1} x_{k}
$$

for every $k=1,2, \ldots, n-1$. Therefore,

$$
x_{k} x_{k}^{-1}=\left(z_{k-1} t_{i_{k}}^{\epsilon_{k}}\right)^{-1}\left(z_{k-1} t_{i_{k}}^{\epsilon_{k}}\right)=t_{i_{k}}^{-\epsilon_{k}} z_{k-1}^{-1} z_{k-1} t_{i_{k}}^{\epsilon_{k}}=t_{i_{k}}^{-\epsilon_{k}} x_{k-1}^{-1} x_{k-1} t_{i_{k}}^{\epsilon_{k}}=\phi_{i_{k}}^{\epsilon_{k}}\left(x_{k-1}^{-1} x_{k-1}\right) .
$$

for every $k=2,3, \ldots, n$ and also $x_{1} x_{1}^{-1}=\phi_{i_{1}}^{\epsilon_{1}}\left(z_{0}^{-1} z_{0}\right)$. We note that if $\epsilon_{k}=1$, then we have $x_{k-1}^{-1} x_{k-1} \in E\left(A_{i_{k}}\right)$ and that if $\epsilon_{k}=-1$, then we have $x_{k-1}^{-1} x_{k-1} \in E\left(B_{i_{k}}\right)$. Using the equations $z_{k}=z_{k-1} t_{i_{k}}^{\epsilon_{k}} x_{k}(k=1,2, \ldots, n-1)$ and $z_{0}=z_{n-1} t_{i_{n}}^{\epsilon_{n}} x_{n}$, we can inductively derive $z_{k-1}=z_{0} t_{i_{1}}^{\epsilon_{1}} x_{1} t_{i_{2}}^{\epsilon_{2}} x_{2} \cdots t_{i_{k-1}}^{\epsilon_{k-1}} x_{k-1}$, for every $k=2,3, \ldots, n$, and hence, we have $z_{0}=z_{0} t_{i_{1}}^{\epsilon_{1}} x_{1} t_{i_{2}}^{\epsilon_{2}} x_{2} \cdots t_{i_{n-1}}^{\epsilon_{n-1}} x_{n-1} t_{i_{n}} x_{n}$. Set $f=z_{0}^{-1} z_{0}$. Then we have $f=z_{0}^{-1} z_{0} t_{i_{1}}^{\epsilon_{1}} x_{1} t_{i_{2}}^{\epsilon_{2}} x_{2} \cdots t_{i_{n-1}}^{\epsilon_{n-1}} x_{n-1} t_{i_{n}}^{\epsilon_{n}} x_{n}$. Since $x_{k} x_{k}^{-1}=\phi_{i_{k}}^{\epsilon_{k}}\left(x_{k-1}^{-1} x_{k-1}\right)$ holds for every $k=2,3, \ldots, n$ and $x_{1} x_{1}^{-1}=\phi_{i_{1}}^{\epsilon_{1}}\left(z_{0}^{-1} z_{0}\right), f$ has a form

$$
z_{0}^{-1} z_{0} t_{i_{1}}^{\epsilon_{1}} x_{1} t_{i_{2}}^{\epsilon_{2}} x_{2} \cdots t_{i_{n-1}}^{\epsilon_{n-1}} x_{n-1} t_{i_{n}}^{\epsilon_{n}} x_{n} .
$$

Since $z_{0}^{-1} z_{0} \in E\left(S^{*}\right)=E(S)$, the form $z_{0}^{-1} z_{0} t_{i_{1}}^{\epsilon_{1}} x_{1} t_{i_{2}}^{\epsilon_{2}} x_{2} \cdots t_{i_{n-1}}^{\epsilon_{n-1}} x_{n-1} t_{i_{n}}^{\epsilon_{n}} x_{n}$ is not reduced by Corollary 3.2 .

We assume that $t_{i_{k}}^{\epsilon_{k}} x_{k} t_{i_{k+1}}^{\epsilon_{k+1}}$ is a pinch for some $k=1,2, \ldots n-1$. There are two possible cases; (1) $i_{k}=i_{k+1}, \epsilon_{k}=-1, \epsilon_{k+1}=1$ and $x_{k} \in A_{i_{k+1}}$ or (2) $i_{k}=i_{k+1}$, $\epsilon_{k}=1, \epsilon_{k+1}=-1$ and $x_{k} \in B_{i_{k+1}}$. We now suppose that case (1) holds. Then we have $\mathbf{y}_{k}=z_{k-1} B_{i_{k}}$ and $\mathbf{y}_{k+1}=z_{k} A_{i_{k+1}}$. We show that $z_{k-1} t_{i_{k}}^{\epsilon_{k}} x_{k} A_{i_{k}}=z_{k-1} t_{i_{k}}^{\epsilon_{k}} A_{i_{k}}$. Note that $\left(z_{k-1} t_{i_{k}}^{\epsilon_{k}}\right)^{-1}\left(z_{k-1} t_{i_{k}}^{\epsilon_{k}}\right)=x_{k} x_{k}^{-1}$ and so $z_{k-1} t_{i_{k}}^{\epsilon_{k}} x_{k} \mathscr{P}, z_{k-1} t_{i_{k}}^{\epsilon_{k}}$. We have

$$
\begin{aligned}
\left(z_{k-1} t_{i_{k}}^{\epsilon_{k}}\right)^{-1}\left(z_{k-1} t_{i_{k}}^{\epsilon_{k}} x_{k}\right) & =x_{k} x_{k}^{-1} x_{k}=x_{k} \in A_{i_{k+1}}=A_{i_{k}}, \\
\left(z_{k-1} t_{i_{k}}^{\epsilon_{k}} x_{k}\right)^{-1}\left(z_{k-1} t_{i_{k}}^{\epsilon_{k}} x_{k}\right) & =x_{k}^{-1}\left(z_{k-1} t_{i_{k}}^{\epsilon_{k}}\right)^{-1}\left(z_{k-1} t_{i_{k}}^{\epsilon_{k}}\right) x_{k} \\
& =x_{k}^{-1} x_{k} x_{k}^{-1} x_{k}=x_{k}^{-1} x_{k} \in E\left(A_{i_{k}}\right)
\end{aligned}
$$

and

$$
\left(z_{k-1} t_{i_{k}}^{\epsilon_{k}}\right)^{-1}\left(z_{k-1} t_{i_{k}}^{\epsilon_{k}}\right)=x_{k} x_{k}^{-1} \in E\left(A_{i_{k}}\right) .
$$

Hence, we have $z_{k-1} t_{i_{k}}^{\epsilon_{k}} x_{k} A_{i_{k}}=z_{k-1} t_{i_{k}}^{\epsilon_{k}} A_{i_{k}}$ by Lemma 4.2. Then we have

$$
\begin{aligned}
\mathbf{y}_{k+1} & =z_{k} A_{i_{k+1}}=z_{k-1} t_{i_{k}}^{\epsilon_{k}} x_{k} A_{i_{k+1}}=z_{k-1} t_{i_{k}}^{\epsilon_{k}} x_{k} A_{i_{k}} \\
& =z_{k-1} t_{i_{k}}^{\epsilon_{k}} A_{i_{k}}=z_{k-1} t_{i_{k}}^{-1} A_{i_{k}}=\overline{z_{k-1} B_{i_{k}}}=\overline{\mathbf{y}_{k}},
\end{aligned}
$$

which contradicts the assumption that there exists no backtracking in the circuit. In the case (2), we can similarly derive a contradiction. Consequently, there exists no circuit in the graph $X$. 


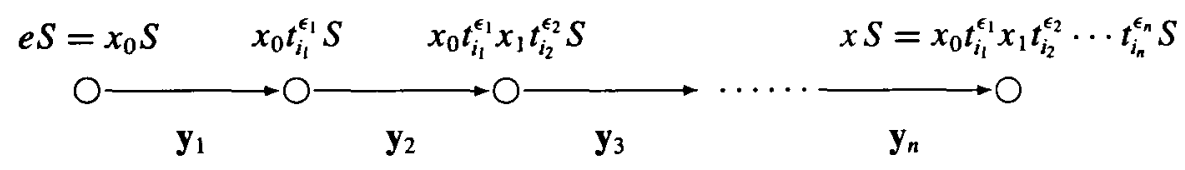

FIGURE 4. Path form $e S$ to $x S$

4.2. Connected component For an idempotent $e \in E\left(S^{*}\right)$, we denote the connected component of $X$ containing the vertex $e S$ by $X_{e}$. We now describe the tree $X_{e}$.

LEMMA 4.5. Let $e$ be an idempotent in $E\left(S^{*}\right)=\xi(S)$. For $x \in S^{*}$, the vertex $x S$ (the edge $x A_{i}$ provided that $x^{-1} x \in E\left(A_{i}\right)$ or the edge $x B_{i}$ provided that $x^{-1} x \in$ $\left.E\left(B_{i}\right)\right)$ is in $X_{e}$ if and only if $x x^{-1}=e$.

PROOF. Suppose that $x x^{-1}=e$. Suppose that $x$ has the normal form $x_{0} t_{i_{1}}^{\epsilon_{1}} x_{1} t_{i_{2}}^{\epsilon_{2}} x_{2} \ldots$ $t_{i_{n-1}}^{\epsilon_{n-1}} x_{n-1} t_{i_{n}}^{\epsilon_{n}} x_{n}$. By Lemma 2.6, we have $e=x x^{-1}=x_{0} x_{0}^{-1}$. By Lemma 4.2, $e S=x_{0} S$ since we have $e, x_{0} \in S$. Similarly, we have $x_{0} t_{i_{1}}^{\epsilon_{1}} x_{1} \cdots t_{i_{k}}^{\epsilon_{k}} S=x_{0} t_{i_{1}}^{\epsilon_{1}} x_{1} \cdots t_{i_{k}}^{\epsilon_{k}} x_{k} S$ for every $k=1,2, \ldots, n$. For each $k=2,3, \ldots, n$, we define $\mathbf{y}_{k}$ to be the edge $x_{0} t_{i_{1}}^{\epsilon_{1}} x_{1} t_{i_{2}}^{\epsilon_{2}} \cdots t_{i_{k-1}}^{\epsilon_{k-1}} x_{k-1} A_{i_{k}}$ if $\epsilon_{k}=1$ and to be the edge $x_{0} t_{i_{1}}^{\epsilon_{1}} x_{1} t_{i_{2}}^{\epsilon_{2}} \cdots t_{i_{k-1}}^{\epsilon_{k-1}} x_{k-1} B_{i_{k}}$ if $\epsilon_{k}=-1$. We define $y_{1}$ to be $x_{0} A_{i_{1}}$ or $x_{0} B_{i_{1}}$ according to $\epsilon_{1}= \pm 1$. Then the path connecting $e S$ and $x S$ is given in Figure 4 .

Note that $x_{0} t_{i_{1}}^{\epsilon_{1}} x_{1} \cdots t_{i_{k}}^{\epsilon_{k}} S=x_{0} t_{i_{1}}^{\epsilon_{1}} x_{1} \cdots t_{i_{k}}^{\epsilon_{k}} x_{k} S$ for every $k=1,2, \ldots, n$. Hence, $x S$ is in the connected component $X_{e}$.

Let $x A_{i}\left(x^{-1} x \in E\left(A_{i}\right)\right)$ be a positively oriented edge such that $x x^{-1}=e$. Suppose that $\mathbf{t}\left(x A_{i}\right)=z S$. Note that $x^{-1} x \leq e_{i}=t_{i} t_{i}^{-1}, \mathbf{i}\left(x A_{i}\right)=x S$ and $\mathbf{t}\left(x A_{i}\right)=x t_{i} S$. Moreover, we have $\left(x t_{i}\right)\left(x t_{i}\right)^{-1}=x t_{i} t_{i}^{-1} x^{-1}=x x^{-1}=e$ since $x^{-1} x \leq t_{i} t_{i}^{-1}$. On the other hand, by Lemma $4.2, z z^{-1}=\left(x t_{i}\right)\left(x t_{i}\right)^{-1}$ since we have $x t_{i} S=z S$. Hence, $z z^{-1}=e$. Similarly, we can show that for a negatively oriented edge $x B_{i}$ such that $\mathbf{t}\left(x B_{i}\right)=z S$ we have $z z^{-1}=e$. It follows that if a vertex $z S$ is connected to a vertex $x S$ such that $x x^{-1}=e$ then we have $z z^{-1}=e$. Since $X_{e}$ is connected, every vertex $z S$ in $X_{e}$ has the property that $z z^{-1}=e$.

We have shown the result for vertices. It is similarly shown that $x A_{i}$ (or $x B_{i}$ ) is in $X_{e}$ if and only if $x x^{-1}=e$.

4.3. Action of a maximal subgroup We now consider a maximal subgroup $S_{e}^{*}$ of $S^{*}$, where $e \in E\left(S^{*}\right)$. Take a vertex $x S$ from $X_{e}$ and $w$ from $S_{e}^{*}$. We have $x x^{-1}=e$ by Lemma 4.5. Then we have

$$
(w x)(w x)^{-1}=w x x^{-1} w^{-1}=w e w^{-1}=w w^{-1}=e
$$

(as $w w^{-1}=w^{-1} w=e$ ). This implies that $w x S$ is in $X_{e}$. Similarly, we can show that $w x A_{i}$ and $w x B_{i}$ are in $X_{e}$ if $x A_{i}$ and $x B_{i}$ are in $X_{e}$. Furthermore, we have 
$\mathbf{I}(s \mathbf{y})=s \mathbf{i}(\mathbf{y}), \mathbf{t}(s \mathbf{y})=s \mathbf{t}(\mathbf{y})$ and $\overline{(s \mathbf{y})}=s \overline{\mathbf{y}}$ for $s \in S_{e}^{*}$ and $\mathbf{y} \in \operatorname{Edge}\left(X_{e}\right)$, that is to say, the group $S_{e}^{*}$ acts on the tree $X_{e}$ from the left. The action preserves the orientation of $X_{e}: S_{e}^{*}$ Edge $_{+}\left(X_{e}\right) \subset$ Edge $_{+}\left(X_{e}\right)$ and $S_{e}^{*}$ Edge $_{-}\left(X_{e}\right) \subset$ Edge $_{-}\left(X_{e}\right)$. Hence, $S_{e}^{*}$ acts on $X_{e}$ without inversion. Denote the orbit of $x S\left(x A_{i}\right.$ and $\left.x B_{i}\right)$ by $\operatorname{Orb}(x S)\left(\operatorname{Orb}\left(x A_{i}\right)\right.$ and $\left.\operatorname{Orb}\left(x B_{i}\right)\right)$. Denote the stabilizer of $x S\left(x A_{i}\right.$ and $\left.x B_{i}\right)$ by $\operatorname{Stab}(x S)\left(\operatorname{Stab}\left(x A_{i}\right)\right.$ and $\left.\operatorname{Stab}\left(x B_{i}\right)\right)$. We describe the orbits and the stabilizers of the vertices and the edges of $X_{e}$.

LEMMA 4.6. Let $x$ be an element in $S^{*}$ such that $x x^{-1}=e$.

(1) We have

$$
\operatorname{Orb}(x S)=\left\{w S \mid w w^{-1}=e, x^{-1} x \mathscr{D}_{S} w^{-1} w\right\} .
$$

(2) If $x^{-1} x \in E\left(A_{i}\right)$, then we have

$$
\operatorname{Orb}\left(x A_{i}\right)=\left\{w A_{i} \mid w w^{-1}=e, w^{-1} w \in E\left(A_{i}\right), x^{-1} x \mathscr{D}_{A_{i}} w^{-1} w\right\} .
$$

(3) If $x^{-1} x \in E\left(B_{i}\right)$, then we have

$$
\operatorname{Orb}\left(x B_{i}\right)=\left\{w B_{i} \mid w w^{-1}=e, w^{-1} w \in E\left(B_{i}\right), x^{-1} x \mathscr{D}_{B_{i}} w^{-1} w\right\} .
$$

Here $\mathscr{D}_{S}, \mathscr{D}_{A_{i}}$ and $\mathscr{D}_{B_{i}}$ denote Green's $\mathscr{D}$-relation of $S, A_{i}$ and $B_{i}$, respectively.

PROOF. Take $w S$ from $\operatorname{Orb}(x S)$. There exists $z$ in $S_{e}^{*}$ such that $z(x S)=w S$. Note that

$$
(z x)^{-1} z x=x^{-1} z^{-1} z x=x^{-1} e x=x^{-1} x x^{-1} x=x^{-1} x .
$$

By Lemma 4.3, we have $z x \mathscr{R} w$ and $(z x)^{-1}(z x) \mathscr{D}_{S} w^{-1} w$. Since $(z x)^{-1} z x=x^{-1} x$, we have $x^{-1} x \mathscr{D}_{S} w^{-1} w$. Moreover, we have

$$
w w^{-1}=(z x)(z x)^{-1}=z x x^{-1} z^{-1}=z e z^{-1}=e .
$$

Hence, we have shown that $w w^{-1}=e$ and $x^{-1} x \mathscr{D}_{S} w^{-1} w$.

Conversely, we assume that $w w^{-1}=e$ and $x^{-1} x \mathscr{D}_{S} w^{-1} w$. There exists $a \in S$ such that $w^{-1} w \mathscr{R}_{s} a \mathscr{L}_{s} x^{-1} x$. Let $d=w a x^{-1}$. Then we have

$$
\begin{aligned}
d d^{-1} & =w a x^{-1}\left(w a x^{-1}\right)^{-1}=w a x^{-1} x a^{-1} w^{-1} \\
& =w a a^{-1} a a^{-1} w^{-1}=w a a^{-1} w^{-1}=w w^{-1} w w^{-1}=w w^{-1}=e
\end{aligned}
$$

and

$$
\begin{aligned}
d^{-1} d & =\left(w a x^{-1}\right)^{-1}\left(w a x^{-1}\right)=x a^{-1} w^{-1} w a x^{-1} \\
& =x a^{-1} a a^{-1} a x^{-1}=x a^{-1} a x^{-1}=x x^{-1} x x^{-1}=e .
\end{aligned}
$$


Hence, $d \in S_{e}^{*}$. We now note that

$$
(w a)(w a)^{-1}=w a a^{-1} w^{-1}=w w^{-1} w w^{-1}=w w^{-1} .
$$

Hence, waR $w$. Since $a \in S$ and $w^{-1} w \in E(S)$, we have $w^{-1} w a \in S$. By Lemma 4.2, we have $w S=w a S$. On the other hand, we have

$$
d x S=w a x^{-1} x S=w a a^{-1} a S=w a S .
$$

Hence, $w S=d x S$ and so $w S \in \operatorname{Orb}(x S)$. It follows that

$$
\operatorname{Orb}(x S)=\left\{w S \mid w w^{-1}=e, x^{-1} x \mathscr{D}_{S} w^{-1} w\right\} .
$$

We can similarly show the other two statements.

LEMMA 4.7. (1) For $x \in S^{*}$ with $x x^{-1}=e$ and $x^{-1} x=f$ we have

$$
\operatorname{Stab}(x S)=x H_{S}(f) x^{-1},
$$

where $H_{S}(f)$ is the group $\mathscr{H}$-class of $S$ containing $f$.

(2) Suppose that $x A_{i}\left(x B_{i}\right)$ is in the tree $X_{e}$. Then we have

$$
\operatorname{Stab}\left(x A_{i}\right)=x H_{A_{i}}(f) x^{-1} \quad\left(\operatorname{Stab}\left(x B_{i}\right)=x H_{B_{i}}(f) x^{-1}\right),
$$

where $H_{A_{i}}(f)\left(H_{B_{i}}(f)\right)$ is the group $\mathscr{H}$-class of $A_{i}\left(B_{i}\right)$ containing $f$.

Proof. We show that $\operatorname{Stab}(x S)=x H_{S}(f) x^{-1}$. Take an element $z$ from $\operatorname{Stab}(x S)$. Then $z x S=x S$. Note that $z z^{-1}=z^{-1} z=e=x x^{-1}$. By Lemma 4.2, we have $z x \mathscr{R} x$ and $x^{-1}(z x) \in S$. Set $g=x^{-1} z x$. Then we have

$$
g g^{-1}=x^{-1} z x\left(x^{-1} z x\right)^{-1}=x^{-1} z x x^{-1} z^{-1} x=x^{-1} z z^{-1} z z^{-1} x=x^{-1} x x^{-1} x=x^{-1} x=f
$$

and

$$
g^{-1} g=\left(x^{-1} z x\right)^{-1} x^{-1} z x=x^{-1} z^{-1} x x^{-1} z x=x^{-1} z^{-1} z z^{-1} z x=x^{-1} x x^{-1} x=x^{-1} x=f .
$$

Hence, $g \in H_{S}(f)$. Then $z=x x^{-1} z x x^{-1}=x g x^{-1} \in x H_{S}(f) x^{-1}$. Thus, $\operatorname{Stab}(x S) \subset$ $x H_{S}(f) x^{-1}$.

Conversely, we take an element $v$ from $H_{S}(f)$. Then we have

$$
\left(x v x^{-1}\right) x S=x v x^{-1} x S=x v f S=x v S
$$

(as $v \in H_{S}(f)$ ). We next show that $x v \mathscr{R} x$ and $x^{-1}(x v) \in S$. Note that

$$
(x v)(x v)^{-1}=x v v^{-1} x^{-1}=x f x^{-1}=x x^{-1} x x^{-1}=x x^{-1} .
$$

Hence, $x v \mathscr{R} x$. Since $x^{-1} x v=f v=v$ (as $v \in H_{S}(f)$ ), we have $x^{-1} x v \in S$ (as $v \in S$ ). By Lemma 4.2, we have $x v S=x S$. It follows that $\left(x v x^{-1}\right) x S=x S$. Hence, $x v x^{-1} \in \operatorname{Stab}(x S)$. This shows that $x H_{S}(f) x^{-1} \subset \operatorname{Stab}(x S)$. Consequently, $\operatorname{Stab}(x S)=x H_{S}(f) x^{-1}$. Similarly it can be shown that $\operatorname{Stab}\left(x A_{i}\right)=x H_{A_{i}}(f) x^{-1}$ and $\operatorname{Stab}\left(x B_{i}\right)=x H_{B_{i}}(f) x^{-1}$. 


\section{Presentation of a maximal subgroup}

Using the action of $S_{e}^{*}$ on $X_{e}$, we obtain a group presentation of $S_{e}^{*}$.

5.1. Graph of groups $S_{e}^{*} \| X_{e}=\left(K, Z_{e}\right) \quad$ We describe the graph of groups induced from the action of $S_{e}^{*}$ on the tree $X_{e}$. Let $S_{e}^{*} \backslash \backslash X_{e}=\left(K, Z_{e}\right)$ be the graph of groups obtained from the action of $S_{e}^{*}$ on $X_{e}$ in the Bass-Serre theory. We recall the construction of $\left(K, Z_{e}\right)$.

(1) Graph $Z_{e}$ The graph $Z_{e}$ is formed by the set of vertices

$$
\operatorname{Vert}\left(Z_{e}\right)=\left\{\operatorname{Orb}(x S) \mid x x^{-1}=e\right\}
$$

and the set of edges

$$
\operatorname{Edge}\left(Z_{e}\right)=\operatorname{Edge}_{+}\left(Z_{e}\right) \cup \operatorname{Edge}_{-}\left(Z_{e}\right)
$$

where

$$
\operatorname{Edge}_{+}\left(Z_{e}\right)=\left\{O \operatorname{Orb}\left(x A_{i}\right) \mid x x^{-1}=e, x^{-1} x \in E\left(A_{i}\right), i \in I\right\}
$$

is the set of positively oriented edges and

$$
\text { Edge }_{-}\left(Z_{e}\right)=\left\{\operatorname{Orb}\left(x B_{i}\right) \mid x x^{-1}=e, x^{-1} x \in E\left(B_{i}\right), i \in I\right\}
$$

is the set of negatively oriented edges. The initial vertex and the terminal vertex of $\operatorname{Orb}\left(x A_{i}\right)$ are $\operatorname{Orb}(x S)$ and $\operatorname{Orb}\left(x t_{i} S\right)$, respectively. The initial vertex and the terminal vertex of $\operatorname{Orb}\left(x B_{i}\right)$ are $\operatorname{Orb}(x S)$ and $\operatorname{Orb}\left(x t_{i}^{-1} S\right)$, respectively. The inverse edge of $\operatorname{Orb}\left(x A_{i}\right)$ is $\operatorname{Orb}\left(x t_{i} B_{i}\right)$ and the inverse edge of $\operatorname{Orb}\left(x B_{i}\right)$ is $\operatorname{Orb}\left(x t_{i}^{-1} A_{i}\right)$. It is easy to verify that $Z_{e}$ forms a graph.

(2) Groups $K$ Let $T$ be a maximal subtree of $Z_{e}$, that is, a spanning tree of $Z_{e}$. There exists a lifting $j: T \rightarrow X_{e}$ because $T$ is a tree (see [14]). We extend $j$ to all edges of $Z_{e}$ so that we can have $j(\mathbf{i}(\mathbf{y}))=\mathbf{i}(j(\mathbf{y}))$ for every $\mathbf{y} \in \operatorname{Edge}_{+}\left(Z_{e}\right)$. Then $j$ is no longer a graph morphism. The groups associated with $\operatorname{Orb}(x S), \operatorname{Orb}\left(x A_{i}\right)$ and $\operatorname{Orb}\left(x B_{i}\right)(i \in I)$ are defined by

and

$$
K_{\operatorname{Orb}(x S)}=\operatorname{Stab}(j(\operatorname{Orb}(x S))), \quad K_{\operatorname{Orb}\left(x A_{i}\right)}=\operatorname{Stab}\left(j\left(\operatorname{Orb}\left(x A_{i}\right)\right)\right)
$$

$$
K_{\mathrm{Orb}\left(x B_{i}\right)}=\operatorname{Stab}\left(j\left(\mathrm{Orb}\left(x B_{i}\right)\right)\right) .
$$

If $s \mathbf{y}=\mathbf{y}$, then $s \overline{\mathbf{y}}=\overline{\mathbf{y}}$ for every $\mathbf{y} \in \operatorname{Edge}\left(X_{e}\right)$. Therefore, $K_{\overline{\operatorname{Orb}\left(x A_{i}\right)}}=K_{\operatorname{Orb}\left(x A_{i}\right)}$ and $K_{\overline{\operatorname{Orb}\left(x B_{i}\right)}}=K_{\mathrm{Orb}\left(x B_{i}\right)}$.

(3) Monomorphisms Suppose that $\mathbf{y}$ is a positively oriented edge of $Z_{e}$. Then $\mathbf{y}=\operatorname{Orb}\left(x A_{i}\right)$ for some $x \in S^{*}$ and $i \in I$ such that $x x^{-1}=e$ and $x^{-1} x \in E\left(A_{i}\right)$. We define group isomorphisms

$$
\sigma_{\mathbf{y}}: K_{\mathbf{y}} \rightarrow K_{\mathrm{i}(\mathbf{y})} \quad \text { and } \quad \tau_{\mathbf{y}}: K_{\mathbf{y}} \rightarrow K_{\mathbf{t}(\mathbf{y})} .
$$


Suppose that $j(\mathbf{y})=x A_{i}$. Then $j(\mathbf{i}(\mathbf{y}))=\mathbf{i}(j(\mathbf{y}))=x S$. We have

$$
\begin{aligned}
K_{\mathbf{i}(\mathbf{y})} & =\operatorname{Stab}(j(\mathbf{i}(\mathbf{y})))=\operatorname{Stab}(\mathbf{i}(j(\mathbf{y}))) \\
& =\operatorname{Stab}\left(\mathbf{i}\left(x A_{i}\right)\right)=\operatorname{Stab}(x S)=x H_{S}\left(x^{-1} x\right) x^{-1} .
\end{aligned}
$$

We also have

$$
K_{\mathbf{y}}=\operatorname{Stab}(j(\mathbf{y}))=\operatorname{Stab}\left(x A_{i}\right)=x H_{A_{i}}\left(x^{-1} x\right) x^{-1} .
$$

Note that $x H_{A_{i}}\left(x^{-1} x\right) x^{-1} \subset x H_{S}\left(x^{-1} x\right) x^{-1}$. Then $\sigma_{\mathrm{y}}: K_{\mathrm{y}} \rightarrow K_{\mathrm{I}(\mathrm{y})}$ is defined to be the inclusion mapping.

Since we have

$$
\operatorname{Orb}(j(\mathbf{t}(\mathbf{y})))=\operatorname{Orb}(\mathbf{t}(j(\mathbf{y})))=\operatorname{Orb}\left(\mathbf{t}\left(x A_{i}\right)\right)=\operatorname{Orb}\left(x t_{i} S\right),
$$

we can choose an element $\gamma_{y}$ from $S_{e}^{*}$ such that

$$
j(\mathbf{t}(\mathbf{y}))=\gamma_{\mathbf{y}} x t_{i} S .
$$

Then we have

$$
\begin{aligned}
K_{\mathbf{t}(\mathbf{y})} & =\operatorname{Stab}(j(\mathbf{t}(\mathbf{y})))=\operatorname{Stab}\left(\gamma_{y} x t_{i} S\right)=\left(\gamma_{y} x t_{i}\right) H_{S}\left(\left(\gamma_{y} x t_{i}\right)^{-1}\left(\gamma_{y} x t_{i}\right)\right)\left(\gamma_{y} x t_{i}\right)^{-1} \\
& =\gamma_{\mathbf{y}} x t_{i} H_{S}\left(t_{i}^{-1} x^{-1} \gamma_{y}^{-1} \gamma_{\mathbf{y}} x t_{i}\right) t_{i}^{-1} x^{-1} \gamma_{y}^{-1}=\gamma_{y} x t_{i} H_{S}\left(\phi_{i}\left(x^{-1} x\right)\right) t_{i}^{-1} x^{-1} \gamma_{y}^{-1}
\end{aligned}
$$

because

$$
t_{i}^{-1} x^{-1} \gamma_{\mathrm{y}}^{-1} \gamma_{\mathrm{y}} x t_{i}=t_{i}^{-1} x^{-1} e x t_{i}=t_{i}^{-1} x^{-1} x x^{-1} x t_{i}=t_{i}^{-1} x^{-1} x t_{i}=\phi_{i}\left(x^{-1} x\right)
$$

(as $x^{-1} x \in E\left(A_{i}\right) \subset A_{i}$ ). Then $\tau_{\mathrm{y}}: K_{\mathrm{y}} \rightarrow K_{\mathrm{t}(\mathrm{y})}$ is defined by

$$
\tau_{\mathbf{y}}(s)=\gamma_{\mathrm{y}} s \gamma_{\mathrm{y}}^{-1}
$$

for $s \in K_{\mathbf{y}}$. For $\mathbf{y} \in$ Edge $_{-}\left(Z_{e}\right)$, we define $\sigma_{\mathrm{y}}$ and $\tau_{\mathrm{y}}$ by $\sigma_{\mathrm{y}}=\tau_{\overline{\mathrm{y}}}$ and $\tau_{\mathrm{y}}=\sigma_{\overline{\mathbf{y}}}$.

5.2. Graph of groups $\left(G, Y_{e}\right)$ We construct a graph of groups $\left(G, Y_{e}\right)$ associated with $S, A_{i}$ and $B_{i}(i \in I)$ for each idempotent $e \in E(S)$ using Green's $\mathscr{D}$-relation. We also show that $\left(G, Y_{e}\right)$ is conjugate isomorphic to the graph of groups $S_{e}^{*} \backslash X_{e}=$ $\left(K, Z_{e}\right)$. Then we can conclude that $S_{e}^{*}$ is isomorphic to the fundamental group of $\left(G, Y_{e}\right)$. Let us start to define the graph of groups $\left(G, Y_{e}\right)$.

(1) Graph $Y$ Let $\operatorname{Vert}(Y)$ be the set of $\mathscr{D}$-classes of $S$, Edge ${ }_{+}(Y)$ be the set of $\mathscr{D}$-classes of $A_{i}$ for all $i \in I$ and Edge $(Y)$ be the set of $\mathscr{D}$-classes of $B_{i}$ for all $i \in I$. We make a convention that Edge ${ }_{+}(Y)$ and Edge $(Y)$ are disjoint although $A_{i}$ may be equal to $B_{j}$ for some $i, j \in I$. We also make a convention that for distinct $i, j \in I$ the set of $\mathscr{D}$-classes of $A_{i}$ is disjoint from the set of $\mathscr{D}$-classes of $A_{j}$ even though $A_{i}$ may coincide with $A_{j}$. We make a similar convention for the set of $\mathscr{D}$-classes of $B_{i}$ 's. The set of edges $\operatorname{Edge}(Y)$ is the union of the set $\operatorname{Edge}_{+}(Y)$ of positively oriented edges and the set Edge_$(Y)$ of negatively oriented edges. Suppose that $D_{A_{i}} \in \operatorname{Edge}_{+}(Y)$, that is, 
$D_{A_{i}}$ is a $\mathscr{D}$-class of $A_{i}$ for some $i \in I$. Then the initial vertex $\mathbf{i}\left(D_{A_{i}}\right)$ is the $\mathscr{D}$-class of $S$ including $D_{A_{i}}$, the terminal vertex $t\left(D_{A_{i}}\right)$ is the $\mathscr{D}$-class of $S$ including $\phi_{i}\left(D_{A_{i}}\right)$ and the inverse edge $\overline{D_{A_{i}}}$ is $\phi_{i}\left(D_{A_{i}}\right)$. Note that $\phi_{i}\left(D_{A_{i}}\right)$ is a $\mathscr{D}$-class of $B_{i}$. Suppose that $D_{B_{i}} \in$ Edge $_{-}(Y)$, that is, $D_{B_{i}}$ is a $\mathscr{D}$-class of $B_{i}$ for some $i \in I$. Then the initial vertex $\mathbf{i}\left(D_{B_{i}}\right)$ is the $\mathscr{D}$-class of $S$ including $D_{B_{i}}$, the terminal vertex $\mathbf{t}\left(D_{B_{i}}\right)$ is the $\mathscr{D}$-class of $S$ including $\phi_{i}^{-1}\left(D_{B_{i}}\right)$ and the inverse edge $\overline{D_{B}}$ is $\phi_{i}^{-1}\left(D_{B_{i}}\right)$. Note that $\phi_{i}^{-1}\left(D_{B_{i}}\right)$ is a $\mathscr{D}$-class of $A_{i}$. It is routine to verify that $Y$ forms a graph in the sense of Serre. We remark that $Y$ is not necessarily connected.

(2) Groups $G$ For each $D_{S} \in \operatorname{Vert}(Y)$, that is, $D_{S}$ is a $\mathscr{D}$-class of $S$, we choose a group $\mathscr{H}$-class of $S$ included in $D_{S}$ and denote it by $G_{D_{S}}$. Similarly, for each $D_{A_{i}} \in$ Edge $_{+}(Y)$, we choose a group $\mathscr{H}$ class of $A_{i}$ included in $D_{A_{i}}$ and denote it by $G_{D_{A_{i}}}$. For each $D_{B_{i}} \in$ Edge $_{-}(Y)$, we set $G_{D_{B_{i}}}=G_{\phi_{i}^{-1}\left(D_{B_{i}}\right)}=G_{\overline{D_{B_{i}}}}$.

(3) Monomorphisms Suppose $D_{A_{i}} \in$ Edge $_{+}(Y)$. There exists a unique $D_{S} \in$ Vert $(Y)$ such that $D_{A_{i}} \subset D_{S}$. Let $K$ be a group $\mathscr{H}$-class of $S$ including $G_{D_{A_{i}}}$. Then $K$ and $G_{D_{s}}$ are group $\mathscr{H}$-classes of $S$ included in $D_{s}$. By Green's lemma there exists $n \in D_{S}$ such that $n K n^{-1}=G_{D_{S}}$. We choose such an element $n$ from $D_{S}$ and fix it. Then $\sigma_{D_{A_{i}}}: G_{D_{A_{i}}} \rightarrow G_{D_{s}}$ is defined by

$$
\sigma_{D_{A_{i}}}(h)=n h n^{-1}
$$

for $h \in G_{D_{A_{i}}}$. We note that $G_{D_{S}}=G_{\left(D_{A_{i}}\right)}$ as $D_{S}=\mathbf{i}\left(D_{A_{i}}\right)$. We now define $\tau_{D_{A_{i}}}: G_{D_{A_{i}}} \rightarrow G_{t\left(D_{A_{i}}\right)}$. There exists a unique $D_{S} \in \operatorname{Vert}(Y)$ such that $\phi_{i}\left(D_{A_{i}}\right) \subset D_{S}$. Let $K$ be the group $\mathscr{H}$-class of $S$ including $\phi_{i}\left(G_{D_{A_{i}}}\right)$. Then $K$ and $G_{D_{S}}$ are group $\mathscr{H}$-classes included in $D_{S}$. By Green's lemma there exists $n \in D_{S}$ such that

$$
n K n^{-1}=G_{D_{s}} .
$$

We choose such an element $n$ from $D_{S}$ and fix it. Then $\tau_{D_{\Lambda_{i}}}: G_{D_{\Lambda_{i}}} \rightarrow G_{D_{S}}$ is defined by

$$
\tau_{D_{A_{i}}}(h)=n \phi_{i}(h) n^{-1}
$$

for $h \in G_{D_{A_{i}}}$. We note that $G_{D_{S}}=G_{\mathbf{t}\left(D_{A_{i}}\right)}$ as $D_{S}=\mathbf{t}\left(D_{A_{i}}\right)$. For $D_{B_{i}} \in$ Edge $_{-}(Y), \sigma_{D_{B_{i}}}$ and $\tau_{D_{B_{i}}}$ are defined by $\sigma_{D_{B_{i}}} \bar{\tau} \tau_{\overline{D_{B_{i}}}}$ and $\tau_{D_{B_{i}}}=\sigma_{\overline{D_{B_{i}}}}$.

(4) Connected component $Y_{e}$ For $e \in E\left(S^{*}\right)=E(S)$, let $Y_{e}$ be the connected component of $Y$ containing the $\mathscr{D}$-class of $S$ containing $e$. We denote the restriction of $(G, Y)$ to $Y_{e}$ by $\left(G, Y_{e}\right)$. Then $\left(G, Y_{e}\right)$ is a connected graph of groups.

5.3. Conjugate isomorphism We prove that $\left(G, Y_{e}\right)$ is conjugate isomorphic to $\left(K, Z_{e}\right)$. The reader is referred to [3] for the definition of a conjugate isomorphism of graphs of groups.

THEOREM 5.1. The graph of groups $\left(K, Z_{e}\right)$ is conjugate isomorphic to the graph of groups $\left(G, Y_{e}\right)$. 
Proof. We define a graph morphism $\Phi: Z_{e} \rightarrow Y_{e}$ by

$$
\Phi(\operatorname{Orb}(x S))=D_{S}\left(x^{-1} x\right), \quad \Phi\left(\operatorname{Orb}\left(x A_{i}\right)\right)=D_{A_{i}}\left(x^{-1} x\right)
$$

and

$$
\Phi\left(\operatorname{Orb}\left(x B_{i}\right)\right)=D_{B_{i}}\left(x^{-1} x\right),
$$

where $D_{S}\left(x^{-1} x\right)\left(D_{A_{i}}\left(x^{-1} x\right)\right.$ and $\left.D_{B_{i}}\left(x^{-1} x\right)\right)$ is the $\mathscr{D}$-class of $S\left(A_{i}\right.$ and $\left.B_{i}\right)$ containing $x^{-1} x$. By Lemma 4.6, $\Phi$ is a well-defined bijection. Note that

$$
\Phi\left(\mathbf{i}\left(\operatorname{Orb}\left(x A_{i}\right)\right)\right)=\Phi(\operatorname{Orb}(x S))=D_{S}\left(x^{-1} x\right)=\mathbf{i}\left(D_{A_{i}}\left(x^{-1} x\right)\right)=\mathbf{i}\left(\Phi\left(\operatorname{Orb}\left(x A_{i}\right)\right)\right) .
$$

Therefore, $\Phi(\mathbf{i}(\mathbf{y}))=\mathbf{i}(\Phi(\mathbf{y}))$ for every $\mathbf{y} \in \operatorname{Edge}_{+}\left(Z_{e}\right)$. Similarly we can show that $\Phi(\mathbf{t}(\mathbf{y}))=\mathbf{t}(\Phi(\mathbf{y}))$ for every $\mathbf{y} \in \operatorname{Edge}_{+}\left(Z_{e}\right)$ and that $\Phi(\mathbf{i}(\mathbf{y}))=\mathbf{i}(\Phi(\mathbf{y}))$ and $\Phi(\mathbf{t}(\mathbf{y}))=\mathbf{t}(\Phi(\mathbf{y}))$ for every $\mathbf{y} \in$ Edge $_{-}\left(Z_{e}\right)$. It is also easy to see that $\Phi(\overline{\mathbf{y}})=\overline{(\Phi(\mathbf{y}))}$ for every $\mathbf{y} \in \operatorname{Edge}\left(Z_{e}\right)$. Thus $\Phi$ is a graph isomorphism of $Z_{e}$ onto $Y_{e}$.

We define isomorphisms between the vertex groups. Let $\mathbf{v}$ be a vertex in $Z_{e}$. Suppose $j(\mathbf{v})=x S$. Then $\mathbf{v}=\operatorname{Orb}(j(\mathbf{v}))=\operatorname{Orb}(x S)$ and so $\Phi(\mathbf{v})=\Phi(\operatorname{Orb}(x S))=$ $D_{S}\left(x^{-1} x\right)$. Then we have

$$
K_{\mathbf{v}}=\operatorname{Stab}(j(\mathbf{v}))=\operatorname{Stab}(x S)=x H_{S}\left(x^{-1} x\right) x^{-1} .
$$

Since $H_{S}\left(x^{-1} x\right)$ and $G_{D_{S}\left(x^{-1} x\right)}$ are group $\mathscr{H}$-classes of $S$ included in $D_{S}\left(x^{-1} x\right)$, there exists $m_{x} \in D_{S}\left(x^{-1} x\right)$ such that

$$
m_{x} H_{S}\left(x^{-1} x\right) m_{x}^{-1}=G_{D_{S}\left(x^{-1} x\right)}
$$

by Green's lemma. We choose such $m_{x}$ and fix it. Then a mapping $\Phi_{\mathrm{v}}: K_{\mathrm{v}} \rightarrow$ $G_{\Phi(v)}=G_{D_{S}\left(x^{-1} x\right)}$ is defined by

$$
\Phi_{\mathbf{v}}(s)=m_{x} x^{-1} s x m_{x}^{-1}
$$

for $s \in K_{\mathrm{v}}=x H_{S}\left(x^{-1} x\right) x^{-1}$. We show that $\Phi_{\mathrm{v}}$ is well defined. Since

$$
x^{-1} s x \in x^{-1} x H_{S}\left(x^{-1} x\right) x^{-1} x=H_{S}\left(x^{-1} x\right),
$$

we have

$$
\Phi_{\mathrm{v}}(s) \in m_{x} H_{S}\left(x x^{-1}\right) m_{x}^{-1}=G_{D_{S}\left(x^{-1} x\right)} .
$$

Note that the choice of $m_{x}$ depends on $x$. We show that $\Phi_{v}$ can be defined so that any other choice of the coset representative $z$ for $x S$ can have an element $m_{z}$ in $D_{S}\left(z^{-1} z\right)$ such that $m_{z} z^{-1} s z m_{z}^{-1}=m_{x} x^{-1} s x m_{x}^{-1}$ for $s \in K_{\mathrm{v}}$. Suppose that $x S=z S$. We define $m_{z}$ to be $m_{x} x^{-1} z$. Then $m_{z}$ belongs to $S$ since $m_{x}$ and $x^{-1} z$ belong to $S$. 
Then $m_{z} \in D_{S}\left(z^{-1} z\right)=D_{S}\left(x^{-1} x\right)$ and $m_{z} z^{-1} s z m_{z}^{-1}=m_{x} x^{-1} z z^{-1} s z\left(m_{x} x^{-1} z\right)^{-1}=$ $m_{x} x^{-1} s x m_{x}^{-1}$. Therefore, $\Phi_{\mathrm{v}}$ is well defined. Clearly $\Phi_{\mathrm{v}}$ is an isomorphism.

We next define isomorphisms between the edge groups. Suppose $\mathbf{y}$ is in $\operatorname{Edge}_{+}\left(Z_{e}\right)$ and $j(\mathbf{y})=x A_{i}$. Then we have

$$
\mathbf{y}=\operatorname{Orb}(j(\mathbf{y}))=\operatorname{Orb}\left(x A_{i}\right)
$$

and so

$$
\Phi(\mathbf{y})=\Phi\left(\operatorname{Orb}\left(x A_{i}\right)\right)=D_{A_{i}}\left(x^{-1} x\right)
$$

We also have

$$
K_{\mathbf{y}}=\operatorname{Stab}(j(\mathbf{y}))=\operatorname{Stab}\left(x A_{i}\right)=x H_{A_{i}}\left(x^{-1} x\right) x^{-1} .
$$

Since $H_{A_{i}}\left(x^{-1} x\right)$ and $G_{D_{A_{i}}\left(x^{-1} x\right)}$ are group $\mathscr{H}$-classes of $A_{i}$ included in $D_{A_{i}}\left(x^{-1} x\right)$, there exists $a_{x} \in D_{A_{i}}\left(x^{-1} x\right)$ such that

$$
a_{x} H_{A_{i}}\left(x^{-1} x\right) a_{x}^{-1}=G_{D_{A_{i}}\left(x^{-1} x\right)}
$$

by Green's lemma. Then a mapping $\Phi_{\mathrm{y}}: K_{\mathbf{y}} \rightarrow G_{\Phi(\mathbf{y})}$ is defined by

$$
\Phi_{y}(s)=a_{x} x^{-1} s x a_{x}^{-1}
$$

for $s \in K_{\mathrm{y}}=x^{-1} H_{A}\left(x x^{-1}\right) x$. For any $z$ such that $x A_{i}=z A_{i}$ we can choose $a_{z}$ from $D_{A_{i}}\left(z^{-1} z\right)=D_{A_{i}}\left(x^{-1} x\right)$ such that $a_{z} z^{-1} s z a_{z}^{-1}=a_{x} x^{-1} s x a_{x}^{-1}$. Therefore $\Phi_{y}$ is well defined. Clearly $\Phi_{\mathbf{y}}$ is an isomorphism. For any edge $\mathbf{y}$ in $\operatorname{Edge}_{-}\left(Z_{e}\right)$, we define $\Phi_{\overline{\mathbf{y}}}$ to be $\Phi_{\mathbf{y}}$.

We now prove that $\Phi$ is a conjugate isomorphism of $\left(K, Z_{e}\right)$ onto $\left(G, Y_{e}\right)$. Recall that $\Phi$ is a conjugate isomorphism if for every edge $\mathbf{y} \in \operatorname{Edge}\left(Z_{e}\right)$ the diagram

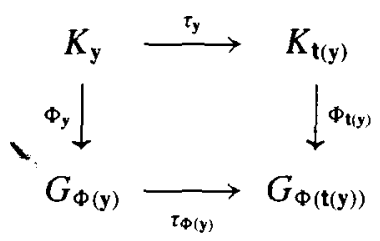

is commutative up to conjugation by an element of $G_{\mathbf{t}(\Phi(\mathbf{y}))}=G_{\Phi(\mathbf{t}(\mathbf{y}))}$.

There are two cases: (1) $\mathbf{y} \in$ Edge $_{+}\left(Z_{e}\right)$ and (2) $y \in$ Edge $_{-}\left(Z_{e}\right)$. We prove the first case and omit the second case.

Suppose that $\mathbf{y}$ is in $\operatorname{Edge}_{+}\left(Z_{e}\right)$ and $j(\mathbf{y})=x A_{i}$. Then $\mathbf{y}=\operatorname{Orb}(j(\mathbf{y}))=\operatorname{Orb}\left(x A_{i}\right)$. We have

$$
K_{\mathbf{y}}=\operatorname{Stab}(j(\mathbf{y}))=\operatorname{Stab}\left(x A_{i}\right)=x H_{A_{i}}\left(x^{-1} x\right) x^{-1} .
$$


Recall that $\gamma_{y}$ satisfies the equation $j(t(y))=\gamma_{y} x t_{i} S$ by (5.1). Then we have

since

$$
\begin{aligned}
K_{\mathbf{t}(\mathbf{y})} & =\operatorname{Stab}(j(\mathbf{t}(\mathbf{y})))=\operatorname{Stab}\left(\gamma_{\mathbf{y}} x t_{i} S\right) \\
& =\gamma_{\mathbf{y}} x t_{i} H_{S}\left(\left(\gamma_{\mathbf{y}} x t_{i}\right)^{-1}\left(\gamma_{\mathbf{y}} x t_{i}\right)\right)\left(\gamma_{\mathbf{y}} x t_{i}\right)^{-1}=\gamma_{\mathbf{y}} x t_{i} H_{S}\left(\phi_{i}\left(x^{-1} x\right)\right)\left(\gamma_{\mathbf{y}} x t_{i}\right)^{-1},
\end{aligned}
$$

$$
\left(\gamma_{y} x t_{i}\right)^{-1}\left(\gamma_{\mathrm{y}} x t_{i}\right)=t_{i}^{-1} x^{-1} \gamma_{\mathrm{y}}^{-1} \gamma_{\mathrm{y}} x t=t_{i}^{-1} x^{-1} x t=\phi_{i}\left(x^{-1} x\right)
$$

Then $\tau_{\mathbf{y}}: K_{\mathbf{y}} \rightarrow K_{\mathrm{t}(\mathrm{y})}$ is defined to be the mapping $s \mapsto \gamma_{\mathrm{y}} s \gamma_{\mathbf{y}}^{-1}$ for $s \in K_{\mathbf{y}}$ by (5.2) and $\Phi_{y}: K_{y} \rightarrow G_{\Phi(y)}$ is defined to be the mapping $s \mapsto a x^{-1} s x a^{-1}$ for $s \in K_{y}$ by (5.9). Note that $a=a_{x}$ is the element of $D_{A_{i}}\left(x^{-1} x\right)$ defined in (5.8). Hence, we have $a_{x} H_{A_{i}}\left(x^{-1} x\right) a_{x}^{-1}=G_{D_{A_{i}}\left(x^{-1} x\right)}$. We recall that $\Phi_{\mathbf{t}(\mathrm{y})}: K_{\mathrm{t}(\mathrm{y})} \rightarrow G_{\Phi(\mathrm{t}(\mathrm{y}))}$ is defined to be the mapping $s \mapsto m\left(\gamma_{y} x t_{i}\right)^{-1} s\left(\gamma_{y} x t_{i}\right) m^{-1}$ for $s \in K_{t(y)}$ by (5.7). Note that $m=m_{\gamma_{g} x_{i}}$ is the element of $D_{S}\left(\phi_{i}\left(x^{-1} x\right)\right)$ defined in (5.6). Hence, we have $m H_{S}\left(\phi_{i}\left(x^{-1} x\right)\right) m^{-1}=G_{D_{S}\left(\phi_{i}\left(x^{-1} x\right)\right)}$. Note that

$$
\Phi(\mathrm{t}(\mathrm{y}))=\Phi\left(\operatorname{Orb}\left(x t_{i} S\right)\right)=D_{S}\left(\left(x t_{i}\right)^{-1}\left(x t_{i}\right)\right)=D_{S}\left(t_{i}^{-1} x^{-1} x t_{i}\right)=D_{S}\left(\phi_{i}\left(x^{-1} x\right)\right) .
$$

We also recall that $\tau_{\Phi(y)}: G_{\Phi(y)} \rightarrow G_{\mathbf{t}(\Phi(y))}$ is defined to be the mapping $s \mapsto n \phi_{i}(s) n^{-1}$ for $s \in G_{\Phi(y)}$ by (5.5). Note that $n$ is the element of $D_{S}\left(\phi_{i}\left(x^{-1} x\right)\right)$ defined by (5.4). Hence, we have $n K n^{-1}=G_{D_{S}\left(\phi\left(x^{-1} x\right)\right)}$, where $K$ is the group $\mathscr{H}$-class of $S$ including $\phi_{i}\left(G_{D_{A_{i}}\left(x^{-1} x\right)}\right)$. See Figure 5 for the $\mathscr{D}$-structure and the location of the elements $m, n, a$.

Take an element $s$ from $H_{A_{i}}\left(x^{-1} x\right)$. Then $x s x^{-1} \in x H_{A_{i}}\left(x^{-1} x\right) x^{-1}=K_{y}$. We have

$$
\begin{aligned}
\Phi_{\mathrm{t}(\mathrm{y})}\left(\tau_{\mathrm{y}}\left(x s x^{-1}\right)\right) & =\Phi_{\mathrm{t}(\mathrm{y})}\left(\gamma_{\mathrm{y}} x s x^{-1} \gamma_{\mathrm{y}}^{-1}\right)=m\left(\gamma_{y} x t_{i}\right)^{-1}\left(\gamma_{y} x s x^{-1} \gamma_{\mathrm{y}}^{-1}\right)\left(\gamma_{\mathrm{y}} x t_{i}\right) m^{-1} \\
& =m t_{i}^{-1} x^{-1} x s x^{-1} x t_{i} m^{-1}=m t_{i}^{-1} s t_{i} m^{-1}=m \phi_{i}(s) m^{-1}
\end{aligned}
$$

On the other hand, we have

$$
\begin{aligned}
\tau_{\Phi(y)}\left(\Phi_{y}\left(x s x^{-1}\right)\right) & =\tau_{\Phi(y)}\left(a x^{-1} x s x^{-1} x a^{-1}\right)=\tau_{\Phi(y)}\left(a s a^{-1}\right) \\
& =n \phi_{i}\left(a s a^{-1}\right) n^{-1}=n \phi_{i}(a) \phi_{i}(s)\left(n \phi_{i}(a)\right)^{-1} .
\end{aligned}
$$

Then we have

$$
\begin{aligned}
n \phi_{i}(a) m^{-1} \Phi_{\mathrm{t}(\mathrm{y})}\left(\tau_{\mathrm{y}}\left(x s x^{-1}\right)\right)\left(n \phi_{i}(a) m^{-1}\right)^{-1} \\
\quad=n \phi_{i}(a) m^{-1}\left(m \phi_{i}(s) m^{-1}\right)\left(n \phi_{i}(a) m^{-1}\right)^{-1} \\
=n \phi_{i}(a) \phi_{i}(s)\left(n \phi_{i}(a)\right)^{-1}=\tau_{\Phi(y)}\left(\Phi_{\mathrm{y}}\left(x s x^{-1}\right)\right) .
\end{aligned}
$$

We prove that $n \phi_{i}(a) m^{-1} \in G_{D_{s}\left(\phi_{i}\left(x^{-1} x\right)\right)}$. By our choice of $n$ and $a$, the element $n^{-1} n$ is the identity of $K$ and the element $\phi_{i}(a) \phi_{i}\left(a^{-1}\right)$ is the identity of $\phi_{i}\left(G_{D_{A_{i}}\left(x^{-1} x\right)}\right)$. Since $\phi_{i}\left(G_{D_{A_{i}}\left(x^{-1} x\right)}\right) \subset K$, we have $n^{-1} n=\phi_{i}(a) \phi_{i}\left(a^{-1}\right)$. Since 
$a^{-1} a=x^{-1} x, \phi_{i}\left(a^{-1}\right) \phi_{i}(a)=\phi_{i}\left(x^{-1} x\right)$. On the other hand, by the choice of $m$, we have $\phi_{i}\left(x^{-1} x\right) \mathscr{R} m^{-1}$. Hence, we have $m^{-1} m=\phi_{i}\left(x^{-1} x\right)=\phi_{i}\left(a^{-1} a\right)$. Then we have

$$
\begin{aligned}
\left(n \phi_{i}(a) m^{-1}\right)^{-1}\left(n \phi_{i}(a) m^{-1}\right) & =m \phi_{i}\left(a^{-1}\right) n^{-1} n \phi_{i}(a) m^{-1} \\
& =m \phi_{i}\left(a^{-1}\right) \phi_{i}(a) m^{-1}=m m^{-1} m m^{-1}=m m^{-1}
\end{aligned}
$$

and so $n \phi_{i}(a) m^{-1} \mathscr{L} m m^{-1}$. By our choice of $n$ and $m$, both $n n^{-1}$ and $m m^{-1}$ are the identity of $G_{D_{s}\left(\phi_{i}\left(x^{-1} x\right)\right)}$. Therefore, $n n^{-1}=m m^{-1}$, that is, $n \mathscr{R} m$. Then

$$
\begin{aligned}
\left(n \phi_{i}(a) m^{-1}\right)\left(n \phi_{i}(a) m^{-1}\right)^{-1} & =n \phi_{i}(a) m^{-1} m \phi_{i}\left(a^{-1}\right) n^{-1} \\
& =n \phi_{i}(a) \phi_{i}\left(a^{-1}\right) \phi_{i}(a) \phi_{i}\left(a^{-1}\right) n^{-1} \\
& =n \phi_{i}(a) \phi_{i}\left(a^{-1}\right) n^{-1}=n n^{-1} n n^{-1}=n n^{-1}=m m^{-1}
\end{aligned}
$$

It follows that $n \phi_{i}(a) m^{-1} \mathscr{R} m m^{-1}$. Hence, $n \phi_{i}(a) m^{-1} \mathscr{H} m m^{-1}$. This implies that $n \phi_{i}(a) m^{-1}$ is in the $\mathscr{H}$-class of $m m^{-1}$, that is, $G_{D_{S}\left(\phi_{i}\left(x^{-1} x\right)\right)}$. Recall that $G_{D_{S}\left(\phi_{i}\left(x^{-1} x\right)\right)}=$ $G_{\mathbf{t}(\Phi(y))}$. Consequently, for every $s \in H_{A_{i}}\left(x^{-1} x\right)$, the element $\Phi_{t(y)}\left(\tau_{y}\left(x s x^{-1}\right)\right)$ is conjugate to $\tau_{\Phi(y)}\left(\Phi\left(x s x^{-1}\right)\right)$ by the element $n \phi_{i}(a) m^{-1}$ of the group $G_{t(\Phi(y))}$.

THEOREM 5.2. $S_{e}^{*}$ is isomorphic to the fundamental group $\pi\left(G, Y_{e}\right)$.

ProOF. By the Bass-Serre theory, the group $S_{e}^{*}$ is isomorphic to $\pi\left(K, Z_{e}\right)$. On the other hand, $\pi\left(G, Y_{e}\right)$ is isomorphic to $\pi\left(K, Z_{e}\right)$ since $\left(G, Y_{e}\right)$ is conjugate isomorphic to $\left(K, Z_{e}\right)$ (see [3, Lemma 21, page 202]). Therefore, $S_{e}^{*}$ is isomorphic to $\pi\left(G, Y_{e}\right)$.

We remark that the group action of the maximal subgroup on the corresponding tree can be integrated into an inverse semigroup action of $S^{*}$ on the forest $X$. The Bass-Serre theory can be generalized to the class of inverse monoids acting on ordered forests; an inverse monoid acting on an ordered forest is characterized as a fundamental inverse monoid of a certain graph of inverse monoids and vice versa. See [12] for the inverse monoid actions on ordered forests, fundamental inverse monoids and graphs of inverse monoids.

\section{Examples}

EXAMPLE 6.1. Let $S$ be an inverse semigroup. Let $A_{i}$ be an inverse subsemigroup such that $e_{i} \in A_{i} \subset e_{i} S e_{i}$ and $E\left(A_{i}\right)=E\left(e_{i} S e_{i}\right)$ for every $i \in I$, where $e_{i} \in E(S)$. Suppose that $B_{i}$ is an inverse subsemigroup of $S$ such that $e_{i} \in B_{i} \subset e_{i} S e_{i}$ and $E\left(B_{i}\right)=E\left(e_{i} S e_{i}\right)$ for every $i \in I$. Let $\phi_{i}$ be an isomorphism of $A_{i}$ onto $B_{i}$ such that $\phi_{i}(f) \mathscr{D} f$ for all $f \in E\left(A_{i}\right)$ for every $i \in I$. It is easy to see that the graph of groups 


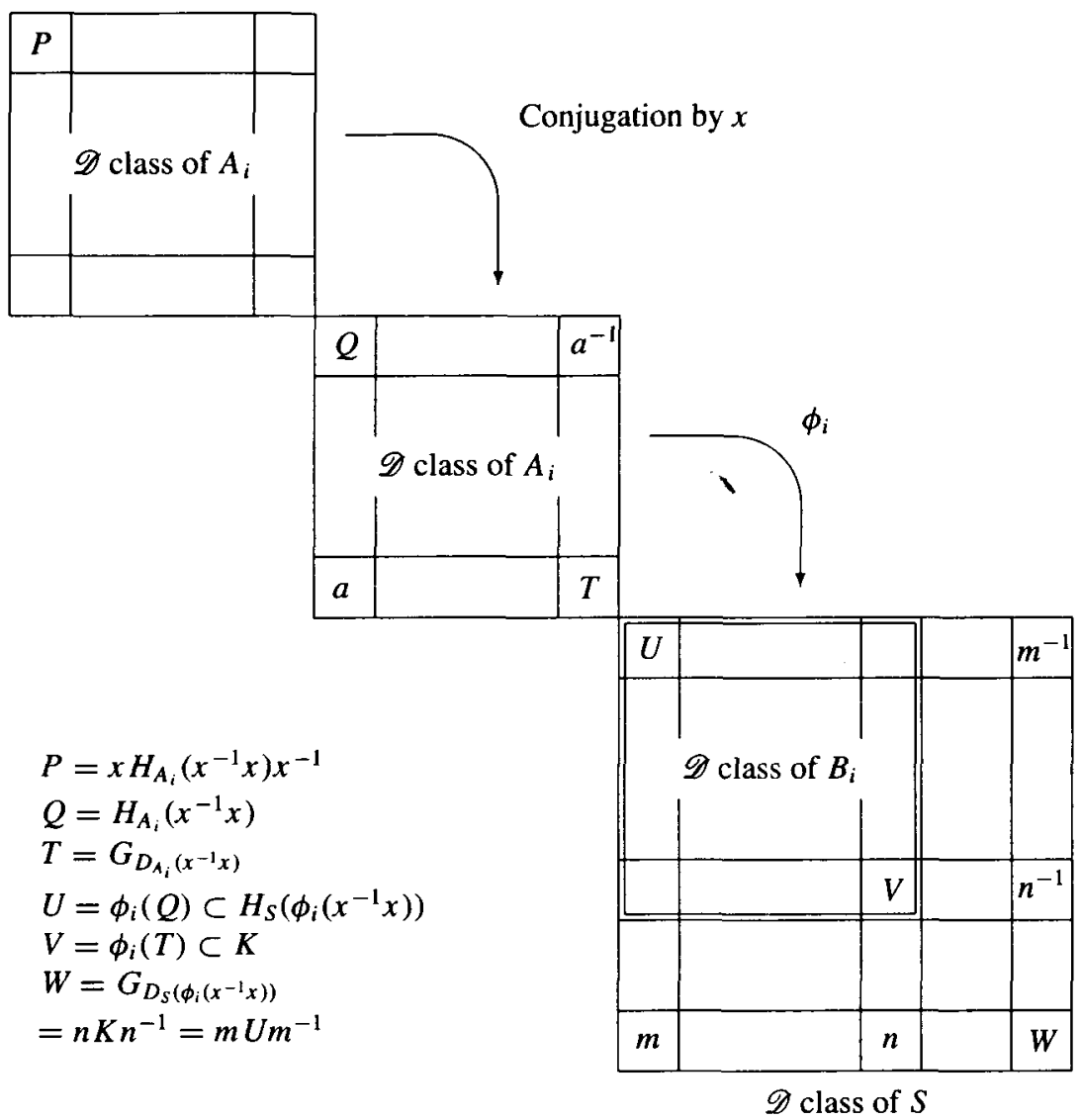

FIGURE 5. $\mathscr{D}$-structure of $S, A_{i}$ and $B_{i}$

$\left(G, Y_{e}\right)$ has the property that $Y_{e}$ is a bouquet, that is, a graph consisting of only one vertex and several edges. It follows that each maximal subgroup of $S^{*}$ is an HNN extension of the maximal subgroup of $S$.

EXAMPLE 6.2. Let $B$ be the bicyclic monoid. Let $\phi$ be the identity mapping on $E(B)$. Let $B^{*}$ be the full HNN extension of $B$ associated with $\phi$. We can conclude that the group $\mathscr{H}$-class $B_{1}^{*}$ of $B^{*}$ is a free group of infinite rank since the graph of groups defined from $B$ is just a bouquet with infinitely many edges such that every vertex group is trivial and the fundamental group of such a graph of groups is a free group generated by infinite generators.

EXAMPLE 6.3. Let $S$ be a strong semilattice $\mathscr{S}\left(E, S_{e}, \rho_{e_{m}}^{e_{n}}\right)$, where $E$ is the $\omega$-chain, 
that is, $E=\left\{e_{0}, e_{1}, e_{2}, \ldots\right\}$ (with the order $e_{m} \leq e_{n}$ if and only if $m \geq n$ ), $S_{e_{n}}$ is a group for every $e_{n} \in E$ and $\rho_{e_{m}}^{e_{n}}: S_{e_{n}} \rightarrow S_{e_{m}}$ is a transition homomorphism for $e_{n}, e_{m} \in E$ with $m \leq n$. Let $A$ be an inverse subsemigroup of $S$ such that $E(S)=E(A)$. Let $B$ be an inverse subsemigroup of $S$ such that $E(B)=E(S) \backslash\left\{e_{0}\right\}$. Suppose that there exists an isomorphism $\phi$ of $A$ onto $B$. Then it is easy to see that the locally full HNN extension $S^{*}$ of $S$ presented by

$$
\operatorname{Inv}\left(S, t \mid t^{-1} a t=\phi(a) \text { for every } a \in A, t^{-1} t=e_{1}, t t^{-1}=e_{0}\right)
$$

is a bisimple regular $\omega$-semigroup, that is, a Reilly extension of a group. The maximal subgroup containing $e_{0}$ is isomorphic to the fundamental group of the graph of groups $\left(G, Y_{e_{0}}\right)$, where $Y_{e_{0}}$ is a half chain, that is, a graph consisting of countably many vertices $\mathbf{v}_{n}$ and countably many edges $\mathbf{y}_{n}$ with $\mathbf{i}\left(\mathbf{y}_{n}\right)=\mathbf{v}_{n-1}$ and $\mathbf{t}\left(\mathbf{y}_{n}\right)=\mathbf{v}_{n}$ for $n=1,2,3, \ldots$ Therefore, $S^{*}$ is a Reilly extension of the fundamental group $\pi\left(G, Y_{e_{0}}\right)$ of the graph of groups $\left(G, Y_{e_{0}}\right)$.

In the case that every $S_{e_{n}}$ is the trivial group, the fundamental group of the graph of groups $\left(G, Y_{e_{0}}\right)$ is trivial. Then, $S^{*}$ is a Reilly extension of the trivial group, that is, $S^{*}$ is the bicyclic semigroup.

EXAMPLE 6.4. Let $S$ be the free Clifford semigroup on a set $X$. Let $E$ be a free semilattice on a set $\left\{e_{x} \mid x \in X\right\}$. For every $e_{x}(x \in X)$, we let $\phi_{x}$ be the identity mapping on the principal ideal $E e_{x}$ generated by $e_{x}$. Clearly, $\phi_{x}$ is an isomorphism of $E e_{x}$ onto itself. It is easy to see that the locally full $\mathrm{HNN}$ extension $E^{*}$ presented by

$$
\operatorname{Inv}\left(E, t_{x}(x \in X) \mid t_{x}^{-1} a t_{x}=\phi_{x}(a)=a \forall a \in E e_{x}, t_{x}^{-1} t_{x}=t_{x} t_{x}^{-1}=e_{x} \forall x \in X\right)
$$

is isomorphic to $S$. Take an idempotent $e$ from $E$. Then $e=e_{x_{1}} e_{x_{2}} \cdots e_{x_{n}}$ for some $x_{1}, x_{2}, \ldots x_{n} \in X$ such that $x_{i}$ 's are distinct each other. We consider the graph of groups $\left(G, Y_{e}\right)$. Clearly, the graph $Y_{e}$ is a bouquet with $n$ edges since all $\phi_{x}$ are identity mappings. Every vertex group is trivial since $E$ is combinatorial. It follows that the fundamental group of $\left(G, Y_{e}\right)$ is a free group of rank $n$. Therefore, the maximal subgroup of the free Clifford semigroup containing the idempotent

$$
e=e_{x_{1}} \boldsymbol{\phi}_{2} \cdots e_{x_{n}}=x_{1} x_{1}^{-1} x_{2} x_{2}^{-1} \cdots x_{n} x_{n}^{-1}
$$

is a free group of rank $n$.

EXAMPLE 6.5. It is known that a universally E-unitary inverse semigroup is a locally full HNN extension of a semilattice of idempotent rank zero ([17]). Hence, a maximal subgroup is a fundamental group of the graph of groups $\left(G, Y_{e}\right)$, where every vertex group $G(\mathbf{v})$ is trivial. This implies that the fundamental group of the graph of group is a free group. Hence, a maximal subgroup of a universally E-unitary inverse semigroup is a free group. The rank of the maximal subgroup can be determined by the shape of the graph $Y_{e}$, in fact, the rank is given by the number of circuits in the graph $Y_{e}$. 


\section{Acknowledgements}

This paper is based on the author's Ph.D. Thesis. The author thanks J. C. Meakin, his thesis advisor, for valuable discussions, advice and encouragement. He is also grateful to the anonymous referee for constructive advice.

\section{References}

[1] P. Bennett, 'On the structure of inverse semigroup amalgams', Int. J. Algebra Comput. (5) 7 (1997), $577-604$.

[2] A. Cherubini, J. C. Meakin and B. Piochi, 'Amalgams of free inverse semigroups', Semigroup Forum 54 (1997), 199-220.

[3] D. E. Cohen, Combinatorial group theory: A topological approach (Cambridge University Press, Cambridge, 1989).

[4] S. Haataja, S. W. Margolis and J. C. Meakin, 'On the structure of full regular semigroup amalgams', J. Algebra 183 (1996), 38-54.

[5] T. E. Hall, 'Free products with amalgamation of inverse semigroups', J. Algebra 34 (1975), 375385.

[6] _ 'Amalgamation of inverse and regular semigroups', Trans. Amer. Math. Soc. 246 (1978), $395-406$.

[7] J. M. Howie, 'Embedding theorems for semigroups', Quart. J. Math. 14 (1963), 254-258.

[8] T. Jajcayova, HNN extensions of inverse semigroups (Ph.D. Thesis, University of NebraskaLincoln, 1997).

[9] M. V. Lawson, Inverse semigroups (World Scientific, Singapore, 1998).

[10] R. C. Lyndon and P. E. Schupp, Combinatorial group theory (Springer, New York, 1976).

[11] S. W. Margolis and J. C. Meakin, 'Inverse monoids, trees and context-free languages', Trans. Amer. Math. Soc. 335 (1993), 259-276.

[12] J. C. Meakin and A. Yamamura, 'Bass-Serre theory and inverse monoids', in: Semigroups and Applications (eds. J. M. Howie and N. Ruškuc) (World Scientific, Singapore, 1998) pp. 125-140.

[13] M. Petrich, Inverse semigroups (Wiley, New York, 1984).

[14] J.-P. Serre, Trees (Springer, New York, 1980).

[15] A. Yamamura, HNN extensions of inverse semigroups (Ph.D. Thesis, University of NebraskaLincoln, 1996).

[16] — 'HNN extensions of inverse semigroups and applications', Int. J. Algebra Comput. (5) 7 (1997), 605-624.

[17] — 'HNN extensions of semilattices', Int. J. Algebra Comput. (5) 9 (1999), 555-596.

[18] _ _ 'Presentations of Bruck-Reilly extensions and decision problems', Semigroup Forum (to appear).

\section{Communications Research Laboratory}

4-2-1, Nukui-Kitamachi, Koganei

Tokyo 184-8795

Japan

e-mail: aki@crl.go.jp 